\title{
An Iterative Approach to Improve Images of Multiple Targets and Targets with Layered or Continuous Profile
}

\author{
Yu-Hsin Kuo and Jean-Fu Kiang \\ Department of Electrical Engineering and the Graduate Institute of Communication Engineering, \\ National Taiwan University, Taipei 106, Taiwan \\ Correspondence should be addressed to Jean-Fu Kiang; jfkiang@ntu.edu.tw
}

Received 4 June 2015; Revised 24 August 2015; Accepted 31 August 2015

Academic Editor: Ramon Gonzalo

Copyright (C) 2015 Y.-H. Kuo and J.-F. Kiang. This is an open access article distributed under the Creative Commons Attribution License, which permits unrestricted use, distribution, and reproduction in any medium, provided the original work is properly cited.

\begin{abstract}
An iterative approach, based on the linear sampling method (LSM) and the contrast source inversion (CSI) method, is proposed to improve the recovered images of multiple targets and targets with layered or continuous profile, including shape and distribution of electric properties. The difficulties in dealing with large targets or high contrast are partly overcome with this approach. Typical targets studied in the literatures are chosen for simulations and comparison.
\end{abstract}

\section{Introduction}

Electromagnetic inverse techniques have been widely explored in geophysical survey, target detection, nondestructive testing, medical imaging, and so forth to retrieve the electric properties of possible targets. The linear sampling method (LSM) has been proposed to estimate the target shape $[1,2]$, implemented with the techniques of singular value decomposition (SVD) and Tikhonov regularization [3]. A level set process (LSP) has also been developed to highlight the target shape more accurately, especially when it has a high conductivity $[4,5]$.

When the target shape is properly acquired, the contrast source inversion (CSI) method can be applied to retrieve the permittivity and conductivity in both the target and the background medium. An IE-CSI (integral equation CSI) method was developed to recover targets immersed in homogeneous or layered background media; and an FD-CSI (finite difference CSI) method was claimed to be suitable for recovering inhomogeneous targets embedded in an inhomogeneous background medium [6-8]. A typical CSI algorithm, facilitated with the SVD technique, usually takes many iterations to converge [1].

The LSM and the CSI method have also been applied to recover multiple targets. For example, nine square cylinders, of width $0.3 \lambda, \epsilon_{r}=2$, and $\sigma=10 \mathrm{mS} / \mathrm{m}$, were placed in free space and probed at $1 \mathrm{GHz}$ [9]. The corners of each cylinder look blurred, and the recovered electric properties appear uneven. It was observed that the permittivity of dielectric targets could be underestimated [10].

In [11], four different inverse methods are compared on the Fresnel dataset [12]. When applying the CSI method to two separated cubes, the dielectric constant in the gap between the cubes is overestimated, especially at lower frequencies. In the case of two jointed spheres, the dielectric constant near the joint is strongly overestimated.

Similarly, in other cases of multiple lossy dielectric cylinders, the electric properties in between cylinders appear to be overestimated, and those near the center of cylinders are underestimated $[13,14]$. When the radius of a cylinder is increased beyond half a wavelength, the dielectric constant near the center of the cylinder is severely underestimated [14].

In [15], a layered target was considered, which is composed of a cylinder of radius $0.15 \lambda, \epsilon_{r}=2$, and $\sigma=0 \mathrm{mS} / \mathrm{m}$, enclosed within a shell of inner radius $0.4 \lambda$, outer radius $0.6 \lambda$, $\epsilon_{r}=1.6$, and $\sigma=0 \mathrm{mS} / \mathrm{m}$. In $[16,17]$, the same geometry was studied, with larger permittivity of both the cylinder and the shell. In all these cases, the permittivity of the shell appears to be underestimated. In [18], two lossy dielectric cylinders were placed within a shell. It was found that the recovered shell image appears to shift inwards, with its dielectric constant being underestimated. On the other hand, the dielectric 


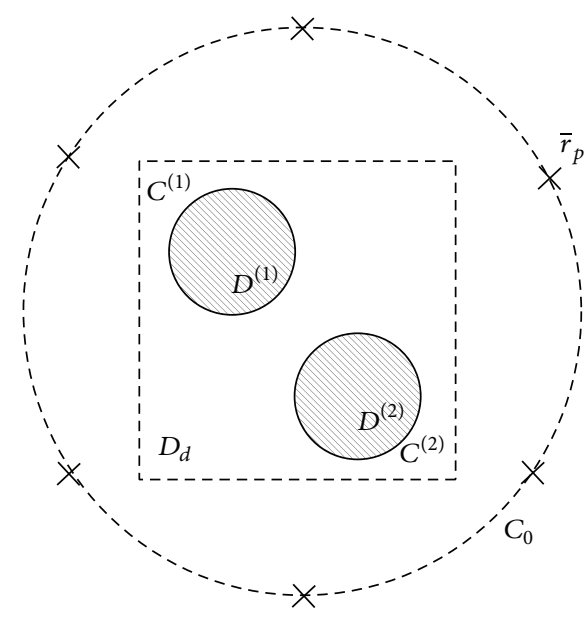

(a)

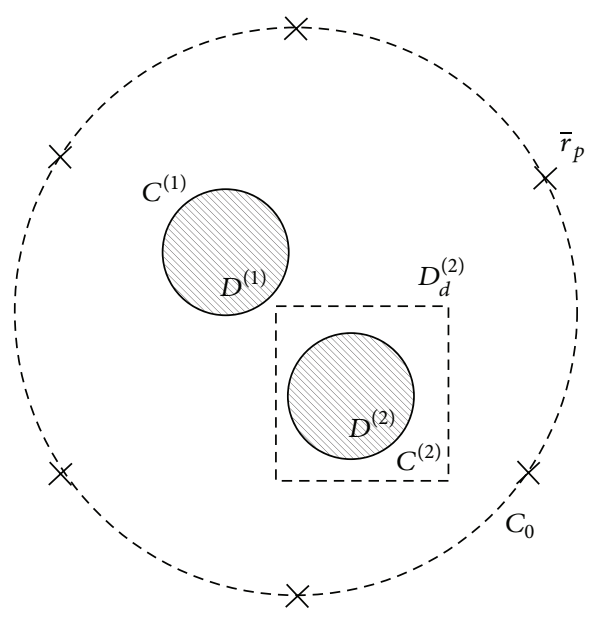

(b)

FIGURE 1: Detection domain (in dashed square) of a two-dimensional inverse problem: (a) the first stage and (b) the second stage.

constant of the two cylinders is overestimated, which may be attributed to the blockage by the shell.

In [19], a coated cube of inner width $0.6 \lambda$ and outer width $1.6 \lambda$, with $\epsilon_{r}=1.6$ and 1.3 , respectively, was considered. The recovered permittivity near the center of the inner cube appears to be underestimated. Similar types of geometry reported in the literatures indicate that the permittivity within a target tends to be underestimated, especially when its electric size is large. With a larger permittivity difference between the target and the background, or between adjacent targets, the recovered permittivity profile becomes less accurate.

In this work, we propose an iterative approach, based on the LSM and the CSI method, to improve the recovered shape and the profile of electric properties of multiple targets, layered targets, and targets with a continuous profile. This paper is organized as follows. The iterative approach is described in Section 2 and simulations results of multiple targets, layered targets, and targets with a continuous profile are presented and discussed in Sections 3, 4, and 5, respectively. Finally, some conclusions are drawn in Section 6.

\section{Description of Iterative Approach}

The inverse process is executed in two stages. In the first stage, apply the linear sampling method (LSM) to estimate the geometrical shape of the target embedded in the detection domain, $D_{d}$, as shown in Figure 1(a). The probes are deployed on the perimeter $C_{0}$, which is outside the detection domain and encloses all the targets. Then, apply the contrast source inversion (CSI) method to estimate the electric parameters in the target domains, $D^{(1)}$ and $D^{(2)}$.

In the second stage, as shown in Figure 1(b), select part of the target domain, $D^{(1)}$, for example, in the first stage and treat it as part of the background. Then, the detection domain is reduced from $D_{d}$ to $D_{d}^{(2)}$; the background field and the scattered field are updated accordingly. Next, apply the LSM to the new detection domain $D_{d}^{(2)}$ to estimate the geometrical shape of the remaining targets; then apply the CSI method to estimate the electric parameters in the remaining target domain.

The LSM and the CSI algorithms are the same as those in [1], which will be briefly described in Sections 2.1 and 2.2. The iterative strategy proposed to improve the accuracy of the target shape and to reduce the rippling artifacts in the inverse results will be presented in Section 2.3.

2.1. LSM in Stage 1. The scattered field is used to estimate the target shape with the LSM [1]. Given an excitation source at $\bar{r}^{\prime \prime}$, the scattered field can be expressed as

$$
\bar{E}_{s}\left(\bar{r}, \bar{r}^{\prime \prime}\right)=k_{b}^{2} \iint_{D_{d}} G\left(\bar{r}, \bar{r}^{\prime}\right) \chi\left(\bar{r}^{\prime}\right) \bar{E}_{t}\left(\bar{r}^{\prime}, \bar{r}^{\prime \prime}\right) d \bar{r}^{\prime}
$$

where $\bar{E}_{s}\left(\bar{r}, \bar{r}^{\prime \prime}\right)$ is the scattered field at $\bar{r}, \bar{E}_{t}\left(\bar{r}^{\prime}, \bar{r}^{\prime \prime}\right)$ is the total field at $\bar{r}^{\prime}, \chi\left(\bar{r}^{\prime}\right)$ is the contrast function of the medium, and $G\left(\bar{r}, \bar{r}^{\prime}\right)$ is the two-dimensional Green's function, which satisfies the wave equation

$$
\left(\nabla^{2}+k_{b}^{2}\right) G\left(\bar{r}, \bar{r}^{\prime}\right)=-\delta\left(\bar{r}-\bar{r}^{\prime}\right) .
$$

It has the explicit form $G\left(\bar{r}, \bar{r}^{\prime}\right)=-(j / 4) H_{0}^{(2)}\left(k_{b}\left|\bar{r}-\bar{r}^{\prime}\right|\right)$, where $H_{0}^{(2)}$ is the zeroth-order Hankel's function of the second kind and $k_{b}$ is the wavenumber of the background medium. The contrast function is defined as

$$
\chi(\bar{r})=\frac{\epsilon(\bar{r})-\epsilon_{b}}{\epsilon_{b}},
$$

where $\epsilon(r)$ and $\epsilon_{b}$ are the complex permittivity of the target and the background medium, respectively. The complex permittivity $\epsilon$ can be expressed as $\epsilon=\epsilon^{\prime}-j \epsilon^{\prime \prime}$, where $\epsilon^{\prime}$ is the real dielectric constant and $\epsilon^{\prime \prime}$ is related to the conductivity $\sigma$ as $\epsilon^{\prime \prime}=\sigma / \omega$. 
Define an adjoint field $\xi\left(\bar{r}, \bar{r}^{\prime \prime}\right)$, which satisfies the adjoint equation

$$
\iint_{D_{d}^{\prime}} \xi\left(\bar{r}, \bar{r}^{\prime \prime}\right) E_{s}\left(\bar{r}^{\prime}, \bar{r}^{\prime \prime}\right) d \bar{r}^{\prime \prime}=G\left(\bar{r}, \bar{r}^{\prime}\right)
$$

where all possible sources are located in $D_{d}^{\prime}$, which is practically outside of $D_{d}$. To solve (4) for $\xi$, consider $M$ excitation probes placed at $\bar{r}_{p n}$ 's, with $1 \leq n \leq M$ [1]. Hence, (4) can be discretized into

$$
\sum_{n=1}^{M} \zeta_{n} \xi\left(\bar{r}, \bar{r}_{p n}\right) E_{s}\left(\bar{r}^{\prime}, \bar{r}_{p n}\right)=G\left(\bar{r}, \bar{r}^{\prime}\right)
$$

where $\zeta_{n}$ is a weighting factor associated with the $n$th excitation probe.

The detection domain $D_{d}$ is divided into $N_{d}$ cells, with the center of the $\ell$ th cell being at $\bar{r}_{\ell}$. For a specific $\bar{r}_{\ell}$, (5) can be discretized into a matrix form as

$$
\overline{\bar{A}} \cdot \bar{f}=\bar{g},
$$

where $A_{m n}=\zeta_{n} E_{s}\left(\bar{r}_{p m}, \bar{r}_{p n}\right), f_{n}=\xi\left(\bar{r}_{\ell}, \bar{r}_{p n}\right)$, and $g_{m}=$ $G\left(\bar{r}_{\ell}, \bar{r}_{p m}\right)$, with $1 \leq m, n \leq M$. Then, apply the singular value decomposition (SVD) and the Tikhonov regularization techniques to solve (6) for $\bar{f}$.

An LSM indicator for cell centered at $\bar{r}_{\ell}$ is calculated as

$$
I_{\xi}\left(\bar{r}_{\ell}\right)=\iint_{D_{d}^{\prime}}\left|\xi\left(\bar{r}_{\ell}, \bar{r}^{\prime \prime}\right)\right|^{2} d \bar{r}^{\prime \prime}=\sum_{n=1}^{M} \zeta_{n}\left|\xi\left(\bar{r}_{\ell}, \bar{r}_{p n}\right)\right|^{2} .
$$

If $I_{\xi}\left(\bar{r}_{\ell}\right)$ is smaller than a threshold value, the cell centered $\bar{r}_{\ell}$ is categorized into part of the target.

2.2. CSI in Stage 1. The right-hand side of (4) can be viewed as an adjoint scattered field, $\Psi_{s}\left(\bar{r}, \bar{r}^{\prime}\right)=G\left(\bar{r}, \bar{r}^{\prime}\right)$, which is the scattered field $\bar{E}_{s}$ operated by $\bar{\xi}$. Similarly, define an adjoint incident field $\Psi_{i}$ and an adjoint total field $\Psi_{t}$ as

$$
\begin{aligned}
\Psi_{i}\left(\bar{r}, \bar{r}^{\prime}\right) & =\iint_{D_{d}^{\prime}} \xi\left(\bar{r}, \bar{r}^{\prime \prime}\right) E_{i}\left(\bar{r}^{\prime}, \bar{r}^{\prime \prime}\right) d \bar{r}^{\prime \prime}, \\
\Psi_{t}\left(\bar{r}, \bar{r}^{\prime}\right) & =\iint_{D_{d}^{\prime}} \xi\left(\bar{r}, \bar{r}^{\prime \prime}\right) E_{t}\left(\bar{r}^{\prime}, \bar{r}^{\prime \prime}\right) d \bar{r}^{\prime \prime} \\
& =\Psi_{i}\left(\bar{r}, \bar{r}^{\prime}\right)+\Psi_{s}\left(\bar{r}, \bar{r}^{\prime}\right) .
\end{aligned}
$$

The last equality holds because $E_{t}=E_{i}+E_{s}$. By substituting (1) into (4) and using (9), we have

$$
G\left(\bar{r}, \bar{r}^{\prime}\right)=k_{b}^{2} \iint_{D_{d}} G\left(\bar{r}, \bar{r}^{\prime \prime}\right) \Psi_{t}\left(\bar{r}^{\prime}, \bar{r}^{\prime \prime}\right) \chi\left(\bar{r}^{\prime \prime}\right) d \bar{r}^{\prime \prime} .
$$

To solve for $\chi\left(\bar{r}^{\prime \prime}\right)$ in the detection domain $D_{d}$, (10) is transformed to a matrix form

$$
\overline{\bar{L}} \cdot \bar{\chi}=\bar{g},
$$

where $\bar{\chi}=\left[\chi\left(\bar{r}_{1}^{\prime \prime}\right), \chi\left(\bar{r}_{2}^{\prime \prime}\right), \ldots, \chi\left(\bar{r}_{N_{d}}^{\prime \prime}\right)\right]^{t}, \bar{g}=\left[G\left(\bar{r}_{p 1}, \bar{r}_{1}^{\prime}\right)\right.$, $G\left(\bar{r}_{p 2}, \bar{r}_{1}^{\prime}\right), \ldots, G\left(\bar{r}_{p M}, \bar{r}_{1}^{\prime}\right), G\left(\bar{r}_{p 1}, \bar{r}_{2}^{\prime}\right), G\left(\bar{r}_{p 2}, \bar{r}_{2}^{\prime}\right), \ldots, G\left(\bar{r}_{p M}, \bar{r}_{2}^{\prime}\right)$ $\left., \ldots, G\left(\bar{r}_{p 1}, \bar{r}_{N_{s}}^{\prime}\right), G\left(\bar{r}_{p 2}, \bar{r}_{N_{s}}^{\prime}\right), \ldots, G\left(\bar{r}_{p M}, \bar{r}_{N_{s}}^{\prime}\right)\right]^{t}$, and

$$
\overline{\bar{L}}=\left[\begin{array}{ccc}
L_{11,1} & \cdots & L_{11, N_{d}} \\
L_{12,1} & \cdots & L_{12, N_{d}} \\
\vdots & \vdots & \vdots \\
L_{1 M, 1} & \cdots & L_{1 M, N_{d}} \\
\vdots & \vdots & \vdots \\
L_{N_{s} M, 1} & \cdots & L_{N_{s} M, N_{d}}
\end{array}\right] .
$$

The explicit form of $L_{n m, \ell}$ is

$$
L_{n m, \ell}=k_{b}^{2} \iint_{\Delta D_{d \ell}} G\left(\bar{r}_{p m}, \bar{r}_{\ell}^{\prime \prime}\right) \Psi_{t}\left(\bar{r}_{n}^{\prime}, \bar{r}_{\ell}^{\prime \prime}\right) d \bar{r}_{\ell}^{\prime \prime},
$$

with $1 \leq n \leq N_{s}, 1 \leq \ell \leq N_{d}, 1 \leq m \leq M$. Similar to the discretization of (4) to derive (5), $\Psi_{t}\left(\bar{r}, \bar{r}^{\prime}\right)$ can be calculated by discretizing (9) as

$$
\Psi_{t}\left(\bar{r}, \bar{r}^{\prime}\right)=\sum_{n=1}^{M} \zeta_{n} \xi\left(\bar{r}, \bar{r}_{p n}\right) E_{t}\left(\bar{r}^{\prime}, \bar{r}_{p n}\right) .
$$

Note that $N_{s}$ is the number of cells in the target domain, which is smaller than $N_{d}$. The SVD and the Tikhonov regularization techniques can then be applied to solve (11) for $\bar{\chi}$.

2.3. LSM and CSI in Stage 2. The definition of scattered field is extended to

$$
\bar{E}_{s}\left(\bar{r}, \bar{r}^{\prime \prime}\right)=\bar{E}_{t}\left(\bar{r}, \bar{r}^{\prime \prime}\right)-\bar{E}_{b}\left(\bar{r}, \bar{r}^{\prime \prime}\right),
$$

where $\bar{E}_{t}\left(\bar{r}, \bar{r}^{\prime \prime}\right)$ is total field and $\bar{E}_{b}\left(\bar{r}, \bar{r}^{\prime \prime}\right)$ is the background field, which is the total field in a given background medium. The background field will reduce to the incident field if the background medium is free space.

A portion of the target area can be selected and merged into the background medium. For example, the shape of $D^{(1)}$, as shown in Figure 1, and the electric parameters are estimated in stage 1 and merged as part of the background. The background field is then numerically calculated and stored as $\bar{E}_{b}^{(1)}\left(\bar{r}, \bar{r}^{\prime \prime}\right)$.

Then, the scattered field $\bar{E}_{s}^{(2)}\left(\bar{r}, \bar{r}^{\prime \prime}\right)$ is updated as

$$
\bar{E}_{s}^{(2)}\left(\bar{r}, \bar{r}^{\prime \prime}\right)=\bar{E}_{t}\left(\bar{r}, \bar{r}^{\prime \prime}\right)-\bar{E}_{b}^{(1)}\left(\bar{r}, \bar{r}^{\prime \prime}\right) .
$$

Based on $\bar{E}_{s}^{(2)}\left(\bar{r}, \bar{r}^{\prime \prime}\right)$, an adjoint field $\xi^{(2)}(\bar{r})$ is defined, which satisfies the adjoint equation

$$
\iint_{D_{d}^{\prime}} \xi^{(2)}\left(\bar{r}, \bar{r}^{\prime \prime}\right) E_{s}^{(2)}\left(\bar{r}^{\prime}, \bar{r}^{\prime \prime}\right) d \bar{r}^{\prime \prime}=G\left(\bar{r}, \bar{r}^{\prime}\right) .
$$

The LSM as used in stage 1 is applied to solve (17) for $\xi^{(2)}$, which is then used to estimate the target shape. 


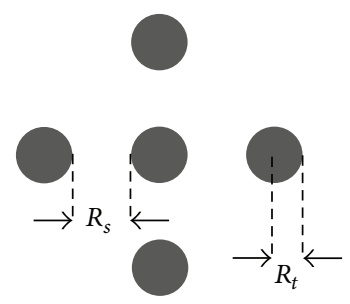

(a)

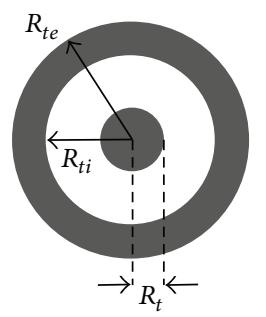

(b)

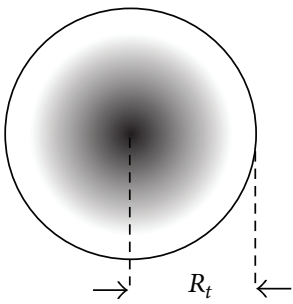

(c)

FiguRE 2: Configurations of (a) five cylinders, (b) a cylinder enclosed by a shell, and (c) a cylinder with continuous permittivity profile.

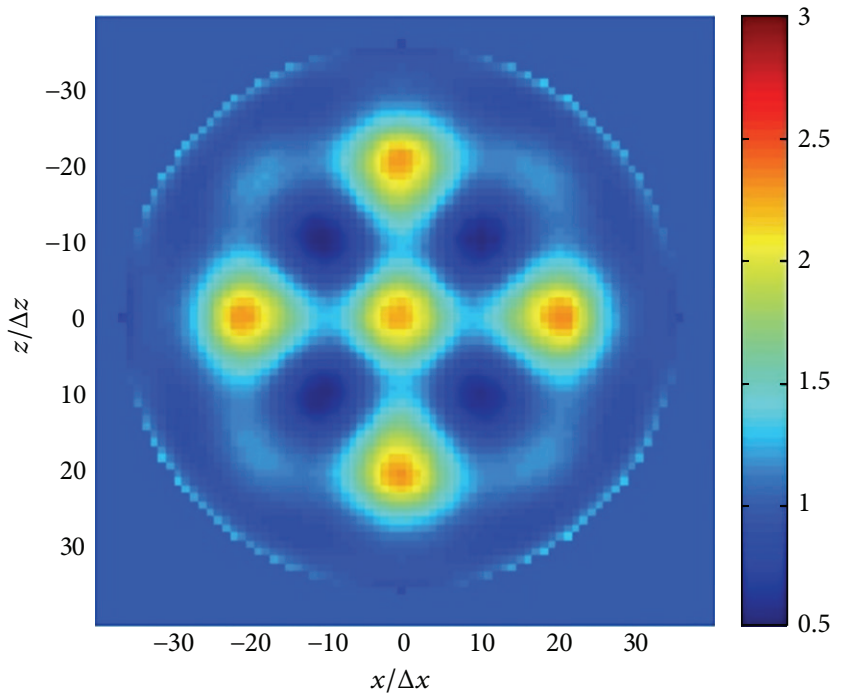

(a)

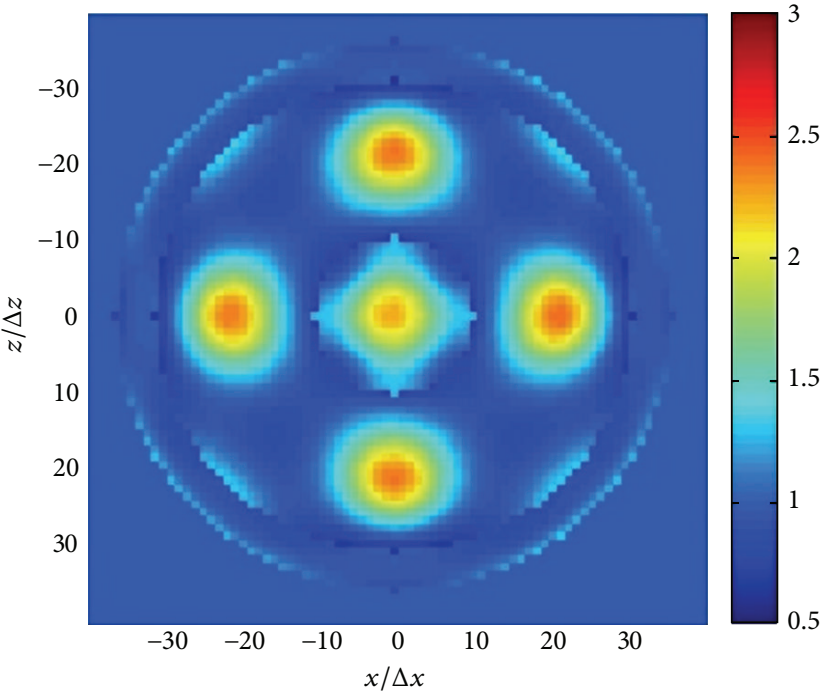

(b)

FiguRE 3: Distribution of relative dielectric constant after (a) stage 1 and (b) stage 2; $R_{t}=0.125 \mathrm{~m}, R_{s}=0.25 \mathrm{~m}, \epsilon_{r}=2.5, \sigma=5 \mathrm{mS} / \mathrm{m}, M=48$, and $R_{d}=0.875 \mathrm{~m}$.

Similar to the CSI method in stage 1, the right-hand side of (17) can be viewed as an adjoint scattered field, $\Psi_{s}^{(2)}\left(\bar{r}, \bar{r}^{\prime}\right)$. The corresponding adjoint incident field $\Psi_{i}^{(2)}$ and adjoint total field $\Psi_{t}^{(2)}$ can be defined as

$$
\begin{aligned}
\Psi_{i}^{(2)}\left(\bar{r}, \bar{r}^{\prime}\right) & =\iint_{D_{d}^{\prime}} \xi^{(2)}\left(\bar{r}, \bar{r}^{\prime \prime}\right) E_{b}^{(1)}\left(\bar{r}^{\prime}, \bar{r}^{\prime \prime}\right) d \bar{r}^{\prime \prime} \\
\Psi_{t}^{(2)}\left(\bar{r}, \bar{r}^{\prime}\right) & =\iint_{D_{d}^{\prime}} \xi^{(2)}\left(\bar{r}, \bar{r}^{\prime \prime}\right) E_{t}\left(\bar{r}^{\prime}, \bar{r}^{\prime \prime}\right) d \bar{r}^{\prime \prime} \\
& =\Psi_{i}^{(2)}\left(\bar{r}, \bar{r}^{\prime}\right)+\Psi_{s}^{(2)}\left(\bar{r}, \bar{r}^{\prime}\right) .
\end{aligned}
$$

Next, substitute (16) into (17) to have

$$
\begin{aligned}
& G\left(\bar{r}, \bar{r}^{\prime}\right) \\
& \quad=k_{b}^{2} \iint_{D_{d}^{(2)}} G\left(\bar{r}, \bar{r}^{\prime \prime}\right) \Psi_{t}^{(2)}\left(\bar{r}^{\prime}, \bar{r}^{\prime \prime}\right) \chi^{(2)}\left(\bar{r}^{\prime \prime}\right) d \bar{r}^{\prime \prime}
\end{aligned}
$$

from which $\chi^{(2)}\left(\bar{r}^{\prime \prime}\right)$ in domain $D_{d}^{(2)}$ can be solved by applying the SVD and Tikhonov regularization techniques as used in stage 1 , where $\Psi_{t}^{(2)}\left(\bar{r}, \bar{r}^{\prime}\right)$ is calculated as

$$
\Psi_{t}^{(2)}\left(\bar{r}, \bar{r}^{\prime}\right)=\sum_{n=1}^{M} \zeta_{n} \xi^{(2)}\left(\bar{r}, \bar{r}_{p n}\right) E_{t}\left(\bar{r}^{\prime}, \bar{r}_{p n}\right) .
$$

\section{Multiple Targets}

Figure 2 shows three types of targets that have been commonly tested in the literatures. The efficacy of the proposed strategy will be studied by simulations on these types in the following three sections, respectively.

Figure 2(a) shows five cylindrical targets placed in free space. The radius of the detection domain is $R_{d}=0.875 \mathrm{~m}$ and $M=48$ probes are used. At the operating frequency of $300 \mathrm{MHz}$, the separation between two adjacent probes is about $0.2 \lambda$. The cell size is $\Delta x=\Delta z=\lambda / 40$, and $N_{s} / N_{d}=$ 0.0210 .

The recovered distributions of permittivity and conductivity after stage 1 are shown in Figures 3(a) and 4(a), respectively. In stage 2 , the domain slightly larger than the center cylinder is selected to be part of the background. The results after stage 2 are shown in Figures 3(b) and 4(b), respectively; and the distributions at $z=0$ are shown in Figure 5. The recovered images after stage 1 and stage 2 look similar. The spacing between the center cylinder and the other four seems to be large enough to allow sufficient probing signals to reach all the five cylinders.

Next, the separation between adjacent cylinders is reduced from $R_{s}=0.25 \mathrm{~m}$ to $R_{s}=0.125 \mathrm{~m}$. The recovered 


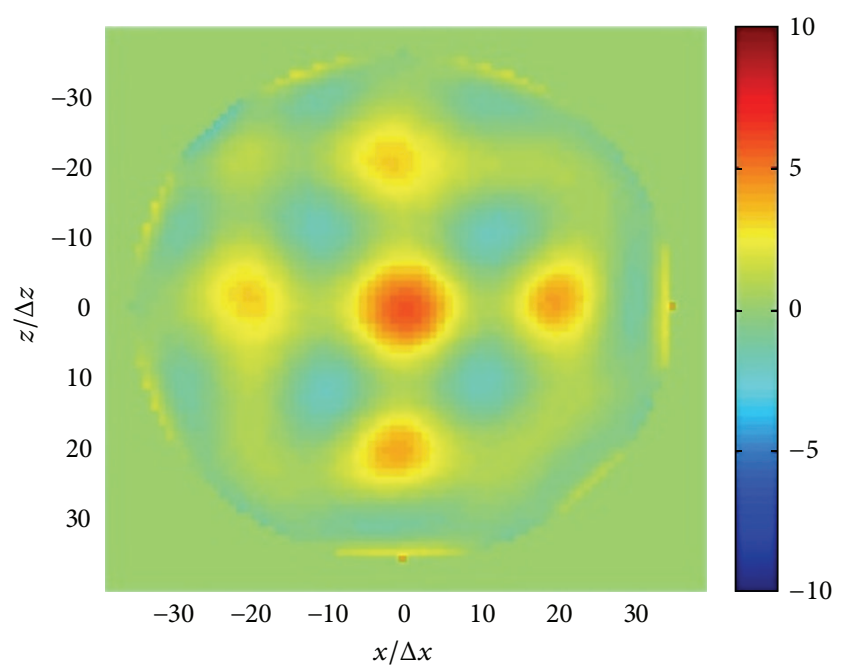

(a)

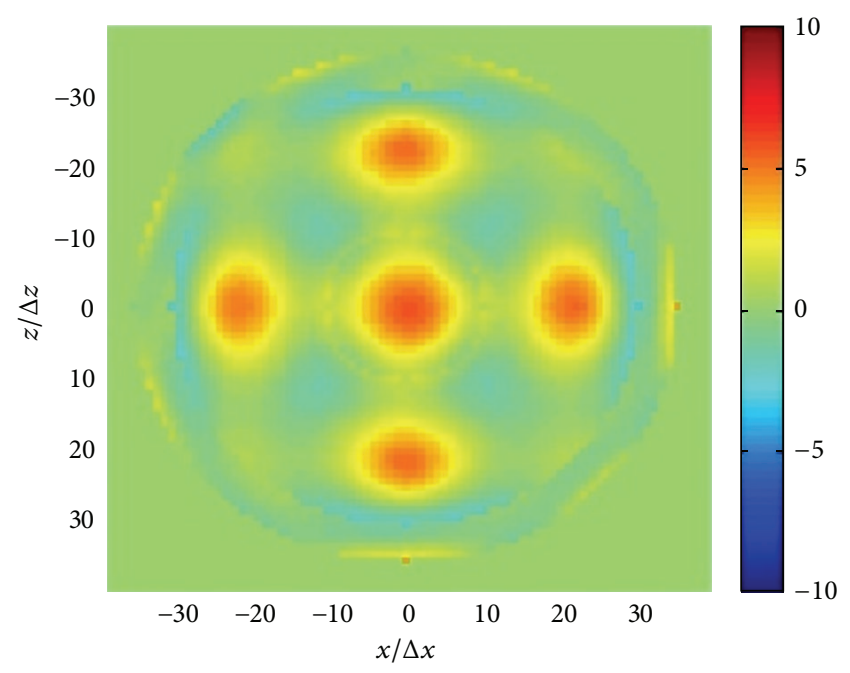

(b)

FIGURE 4: Distribution of conductivity after (a) stage 1 and (b) stage 2; parameters are the same as in Figure 3.

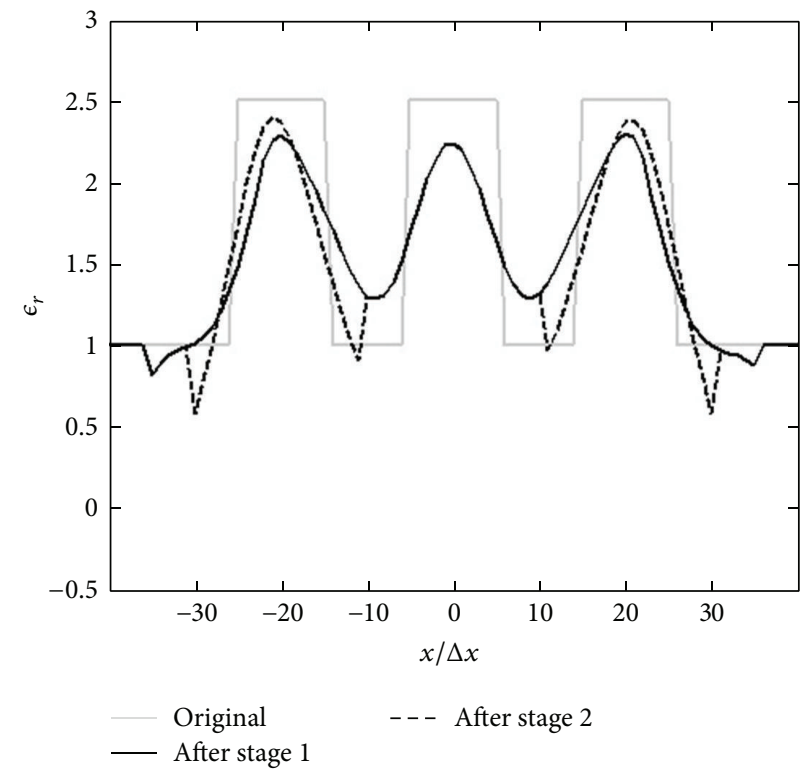

(a)

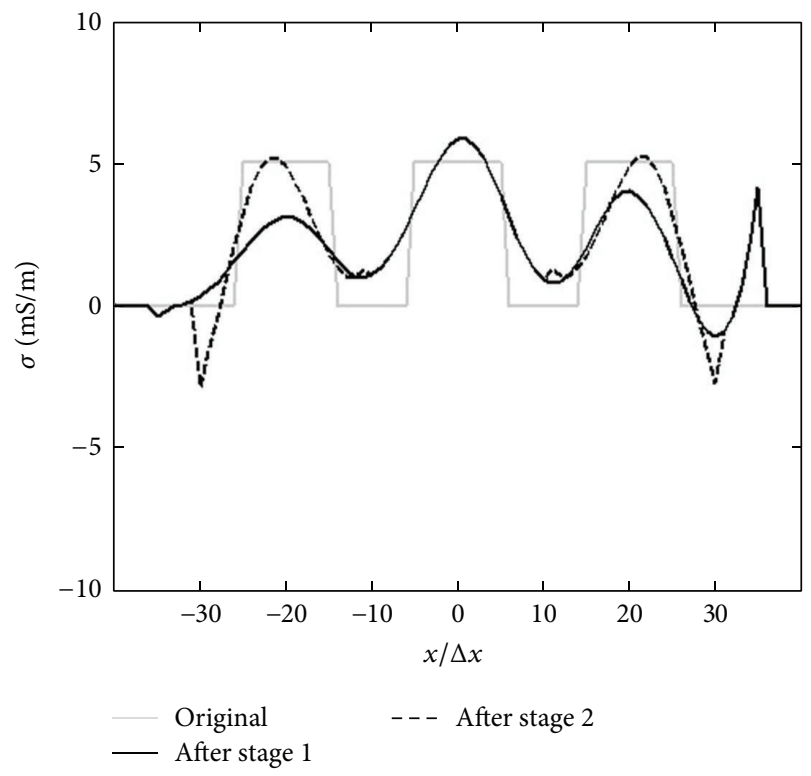

(b)

Figure 5: Distribution of (a) relative dielectric constant and (b) conductivity of multiple targets at $z=0$; parameters are the same as in Figure 3.

permittivity and conductivity distributions after stage 1 are shown in Figures 6(a) and 7(a), respectively. The permittivity in the center cylinder is obviously underestimated.

In stage 2, an annular domain, enclosing the four outer cylinders but excluding the center one, is selected to be part of the background. The results after stage 2 are shown in Figures 6(b) and 7(b), respectively; and the distributions at $z=0$ are shown in Figure 8.

In this case, the separation between the center cylinder and the other four seems to be too small. Some of the probing signals are blocked by the outer four cylinders from reaching the center one. Hence, the permittivity of the center cylinder is underestimated, and that over the gap between the center cylinder and the other four is overestimated.

In summary, when $R_{s}=0.25 \mathrm{~m}$, the results shown in Figures 4 and 5 indicate that both the conventional method and the proposed approach give similar results. When $R_{s}=$ $0.125 \mathrm{~m}$, the results shown in Figures 7 and 8 indicate that the conventional method underestimates the permittivity of the center cylinder because the outer four cylinders block some of the probing waves. After merging the four blocking cylinders to the background, the center cylinder is better observed with the probing waves. 


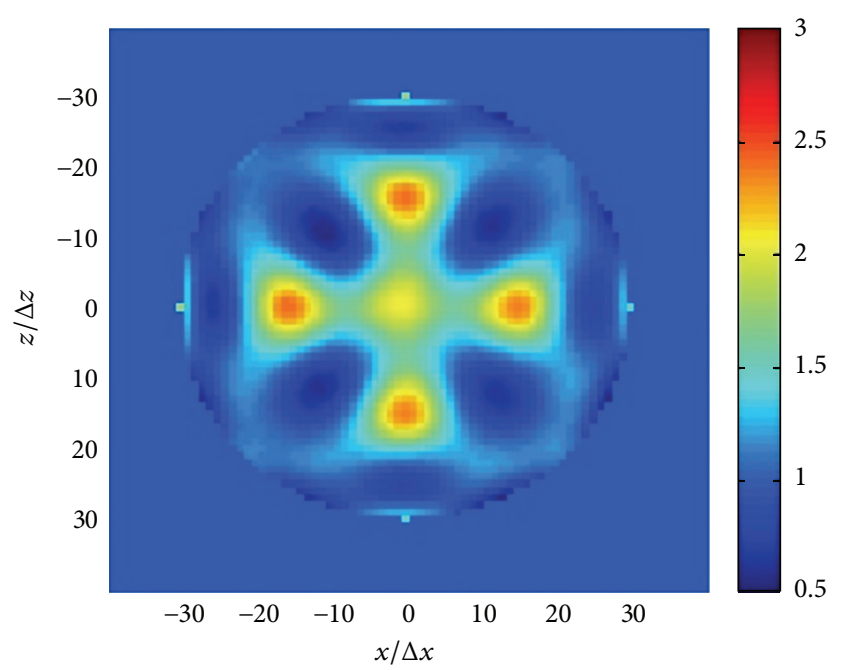

(a)

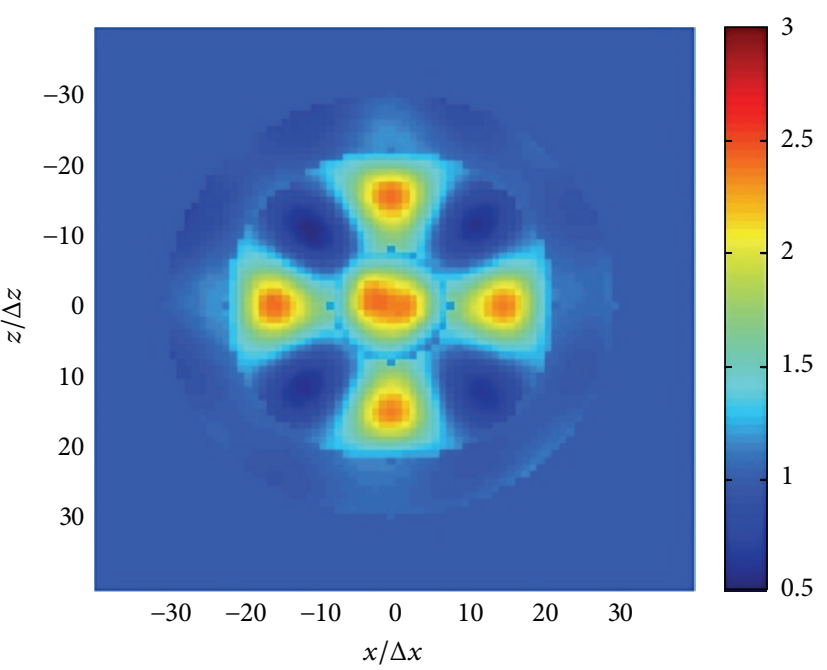

(b)

Figure 6: Distribution of relative dielectric constant after (a) stage 1 and (b) stage $2 ; R_{t}=0.125 \mathrm{~m}, R_{s}=0.125 \mathrm{~m}, \epsilon_{r}=2.5, \sigma=5 \mathrm{mS} / \mathrm{m}$, $M=48$, and $R_{d}=0.875 \mathrm{~m}$.

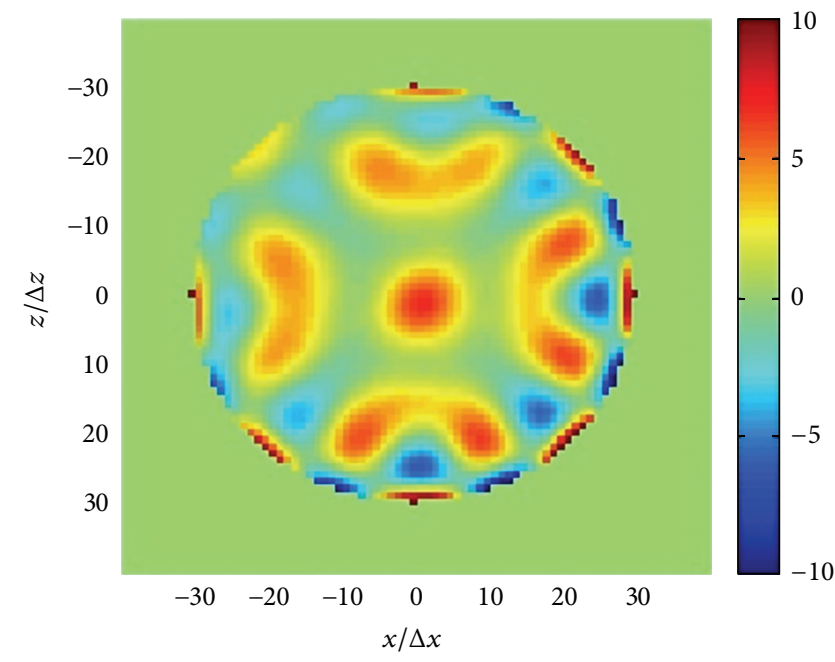

(a)

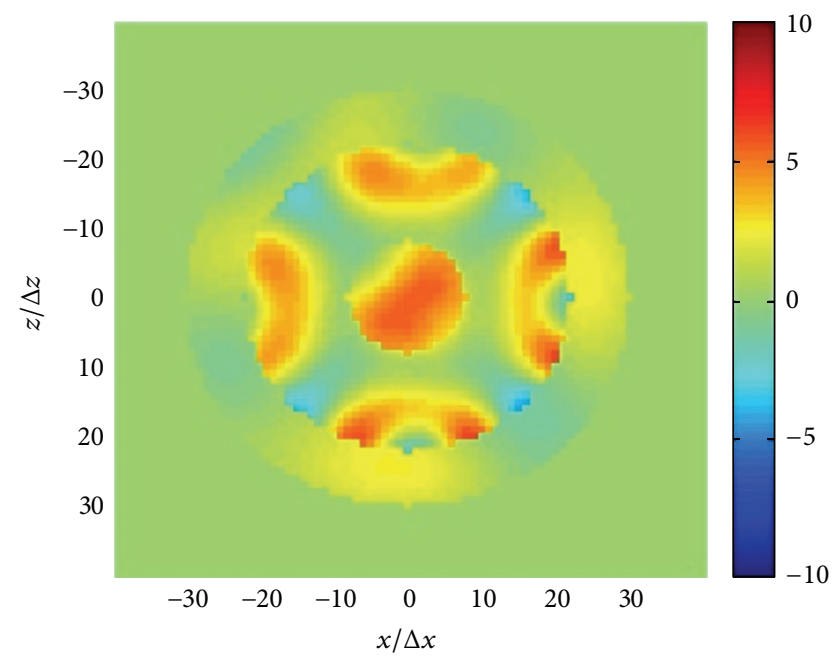

(b)

FIgURE 7: Distribution of conductivity after (a) stage 1 and (b) stage 2; parameters are the same as in Figure 6.

To quantify the improvement of accuracy by using the proposed strategy, we define the error indices on target shape, permittivity, and conductivity as

$$
\begin{aligned}
& \varepsilon_{s}=100 \times \frac{N_{m}}{N_{t}} \%, \\
& \varepsilon_{\epsilon t}=100 \times \sqrt{\frac{\sum_{n=1}^{N_{t}}\left|\epsilon_{t}^{e}-\epsilon_{t n}^{a}\right|}{\sum_{n=1}^{N_{t}}\left|\epsilon_{t n}^{a}\right|} \%,} \\
& \varepsilon_{\sigma t}=100 \times \sqrt{\frac{\sum_{n=1}^{N_{t}}\left|\sigma_{t n}^{e}-\sigma_{t n}^{a}\right|}{\sum_{n=1}^{N_{t}}\left|\sigma_{t n}^{a}\right|} \%,}
\end{aligned}
$$

where $\varepsilon_{s}$ is the shape-error index and $\varepsilon_{\epsilon t}$ and $\varepsilon_{\sigma t}$ are the error indices of permittivity and conductivity, respectively, within the target; $N_{t}$ is the number of cells in the target, $N_{m}$ is the number of cells in the target which are misrecognized as part of the background, and the superscripts $e$ and $a$ indicate the estimated value and the actual value, respectively.

By comparing the results after stages 1 and 2 as shown in Figures 6 and 7, it is observed that $\varepsilon_{s}$ is reduced from $35 \%$ to $30 \%, \varepsilon_{\epsilon t}$ is reduced from $25 \%$ to $23 \%$, and $\varepsilon_{\sigma t}$ is reduced from $48 \%$ to $46 \%$.

\section{Layered Targets}

Figure 2(b) shows a cylinder target enclosed by a cylindrical shell. The cylinder has a radius of $R_{t}=0.1 \mathrm{~m}$, and the shell 


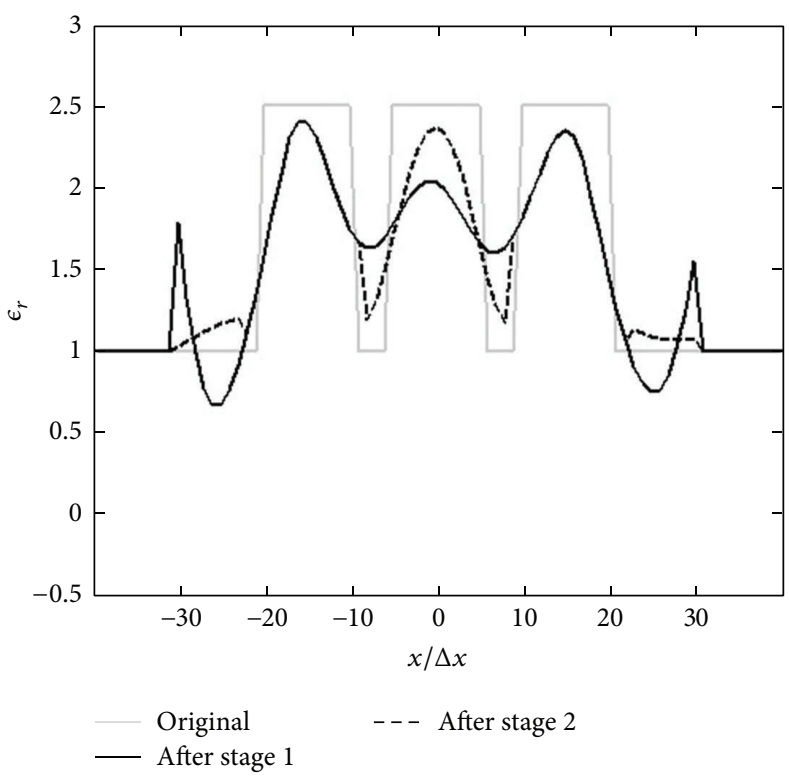

(a)

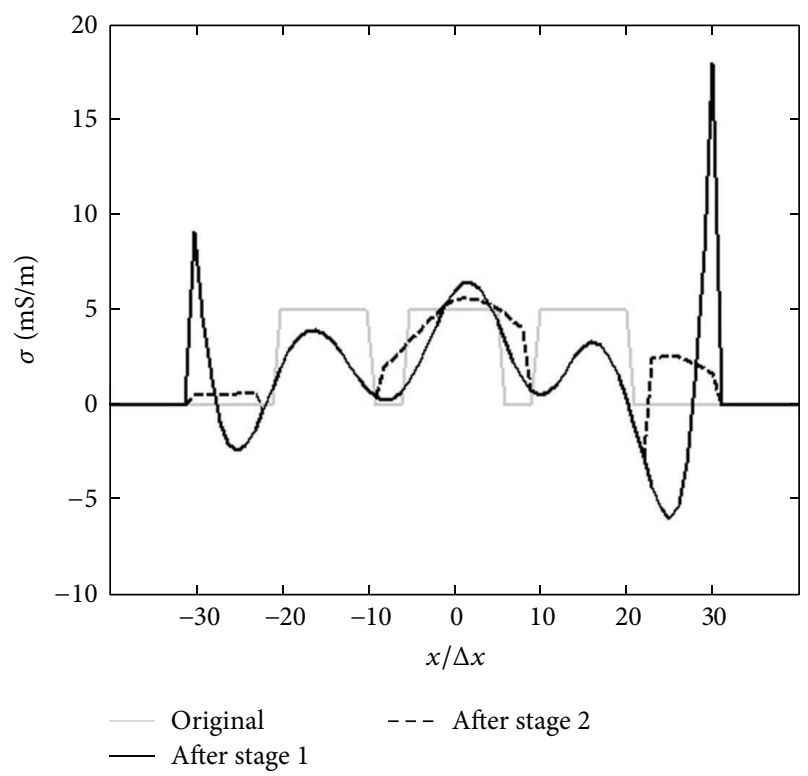

(b)

Figure 8: Distribution of (a) relative dielectric constant and (b) conductivity of multiple targets at $z=0$; parameters are the same as in Figure 6 .

has an internal radius of $R_{t i}=0.3 \mathrm{~m}$ and an external radius of $R_{t e}=0.4 \mathrm{~m}$. The radius of the detection domain is chosen to be $R_{d}=1.25 \mathrm{~m}$, and $M=48$ probes are used. At the operating frequency of $300 \mathrm{MHz}$, the spacing between two adjacent probes is about $0.2 \lambda$. The cell size is $\Delta x=\Delta z=$ $\lambda / 40$.

The solid curves in Figure 9 are the results after stage 1 , by applying the conventional LSM and CSI method. Three different permittivities of target are simulated, and the position of the shell appears to shift inwards in all three cases.

When the iterative approach is applied, the electrical parameters of and around the center cylinder estimated in stage 1 are treated as part of the background medium in stage 2. The recovered distributions at $z=0$ after stage 2 , with $\epsilon_{r}=2.0$ and 2.5, become closer to the original, as compared to the conventional method. For the case with $\epsilon_{r}=3.0$, the permittivity in the shell is underestimated, but the shell position is also closer to the original than that predicted with the conventional method. By comparing the results after stages 1 and 2, it is found that $\varepsilon_{s}$ is reduced from $120 \%$ to $31 \%$, and $\varepsilon_{\epsilon t}$ is reduced from $42 \%$ to $29 \%$.

As the shell is placed too close to the cylinder, the recovered position of the former may be shifted when using the conventional method. Next, we compare the effects of shell-cylinder separation by simulating cases with $\left(R_{t i}, R_{t e}\right)=$ $(0.4,0.5) \mathrm{m}$ and $(0.5,0.6) \mathrm{m}$, with the distributions at $z=0$ shown in Figures 10 and 11, respectively. In both cases, the position shift of shell becomes less severe than the previous case with $\left(R_{t i}, R_{t e}\right)=(0.3,0.4) \mathrm{m}$. The iterative approach not only improves the shell position as in the previous case but also obtains a better estimation of permittivity in the shell.
The thickness of the shell may affect the estimation of shell position and permittivity. Hence, a thicker shell with $\left(R_{t i}, R_{t e}\right)=(0.3,0.5) \mathrm{m}$ is simulated. Figure 12 shows the recovered distributions at $z=0$, where position shift is barely observable.

In summary, by applying the conventional LSM and CSI method, the shell position will be shifted if the cylinder and the shell are put too close or if the shell is too thin. Using the LSM indicator cannot completely separate the shell from the cylinder in some cases. When the shell is thin, more probing waves can reach the internal cylinder and the permittivity of the latter can be well recovered. However, the position of the shell is shifted and its permittivity is underestimated. On the other hand, if the shell is thick, less probing waves can reach the internal cylinder, leading to underestimation of permittivity of the cylinder. The iterative approach seems capable of overcoming this problem by merging the internal cylinder into the background to improve the image of the external shell.

\section{Targets with Continuous Profile}

Figure 2(c) shows a cylinder with a continuous permittivity profile. The radius of the cylinder is $R_{t}=0.375 \mathrm{~m}$, the radius of the detection domain is $R_{d}=1.25 \mathrm{~m}$, and $M=48$ probes are used. At the operating frequency of $300 \mathrm{MHz}$, the spacing between two adjacent probes is about $0.2 \lambda$. The cell size is $\Delta x=\Delta z=\lambda / 40$. Figures 13 and 14 show the recovered distributions of relative dielectric constant and conductivity at $z=0$. The relative dielectric constant has a linear profile, with the maximum $\epsilon_{r}$ of 2.4 and 3.0, respectively. Each profile is approximated as a piecewise-constant function of 4,8 , and 


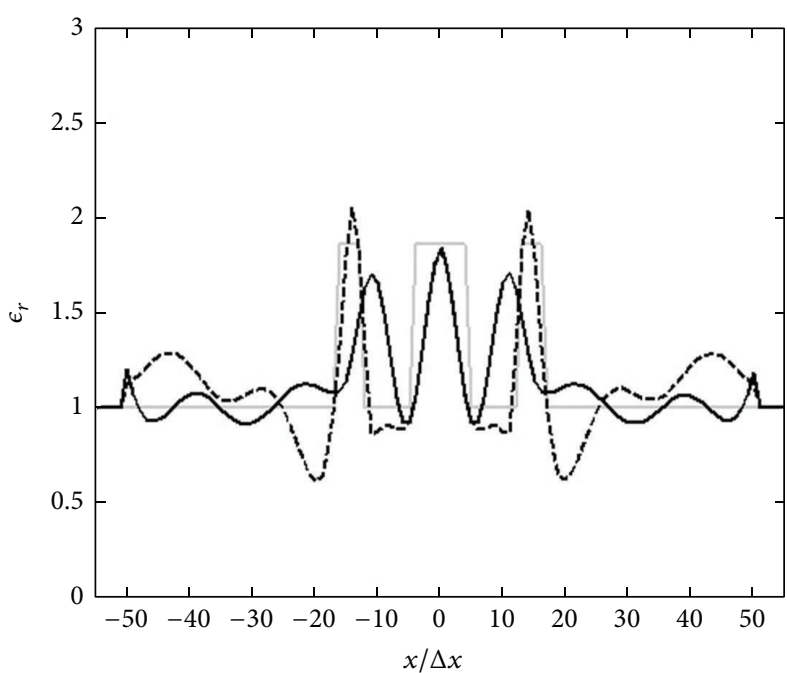

Original Stage 1

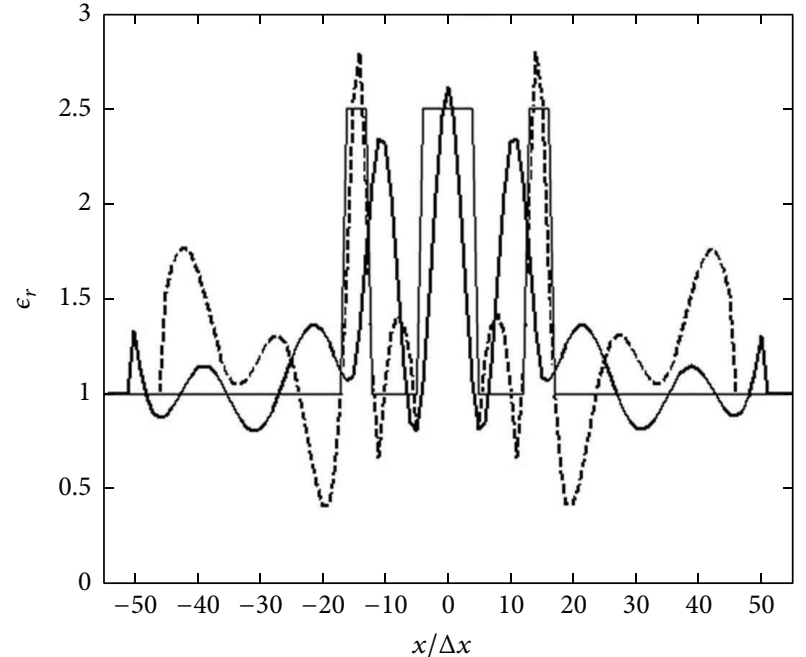

Original - Stage 1

(a)

(b)

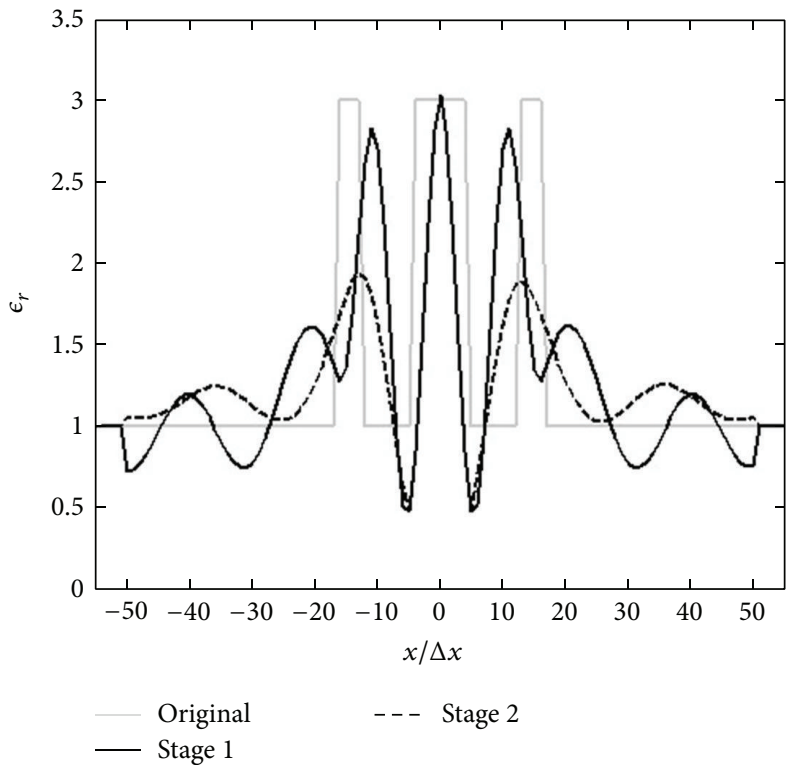

(c)

FiguRE 9: Distribution of relative dielectric constant of layered targets at $z=0$; (a) $\epsilon_{r}=2.0$, (b) $\epsilon_{r}=2.5$, and (c) $\epsilon_{r}=3.0$; $R_{t}=0.1 \mathrm{~m}$, $R_{t i}=0.3 \mathrm{~m}, R_{t e}=0.4 \mathrm{~m}, \sigma=0, M=48$, and $R_{d}=1.25 \mathrm{~m}$.

15 stairs, respectively. The recovered results of the case with $\epsilon_{r, \max }=2.4$ appear closer to the original profile than those with $\epsilon_{r, \max }=3$. As shown in Figure 14, the permittivity in the internal portion of the 4-stair approximation is underestimated.

Figure 15 shows the recovered distributions with $R_{t}=$ $0.375,0.4,0.45$, and $0.5 \mathrm{~m}$, respectively. A linear permittivity profile is assumed, with $\epsilon_{r, \max }=2.4$. With $R_{t}=0.45$ or $0.5 \mathrm{~m}$, the permittivity in the internal portion is seriously underestimated.
Similar recovered images were observed in [20], where two concentric square cylinders were immersed in a background medium with $\epsilon_{r}=1.2$ and $\sigma=5 \mathrm{mS} / \mathrm{m}$ at the operating frequency of $400 \mathrm{MHz}$. The external square cylinder has the width of $0.5 \mathrm{~m}, \epsilon_{r}=3.6$, and $\sigma=50 \mathrm{mS} / \mathrm{m}$. The internal square cylinder has the width of $0.25 \mathrm{~m}, \epsilon_{r}=6$, and $\sigma=80 \mathrm{mS} / \mathrm{m}$. The results using the contrast source-extended Born (CS-EB) approach are consistent with the original, but the results using the contrast source inversion approach of [8] are inconsistent. The permittivity in the internal portion is 


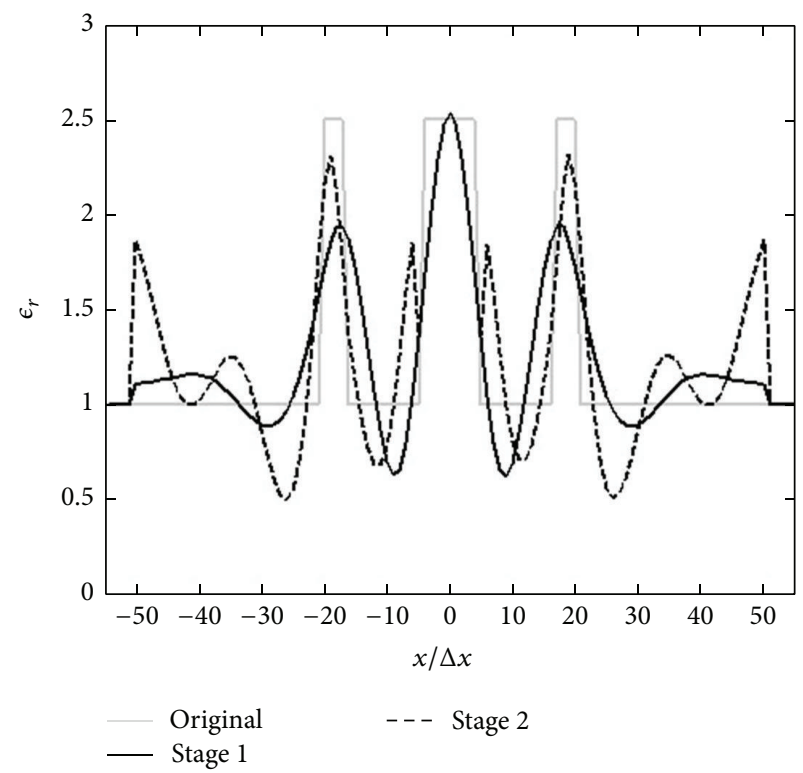

(a)

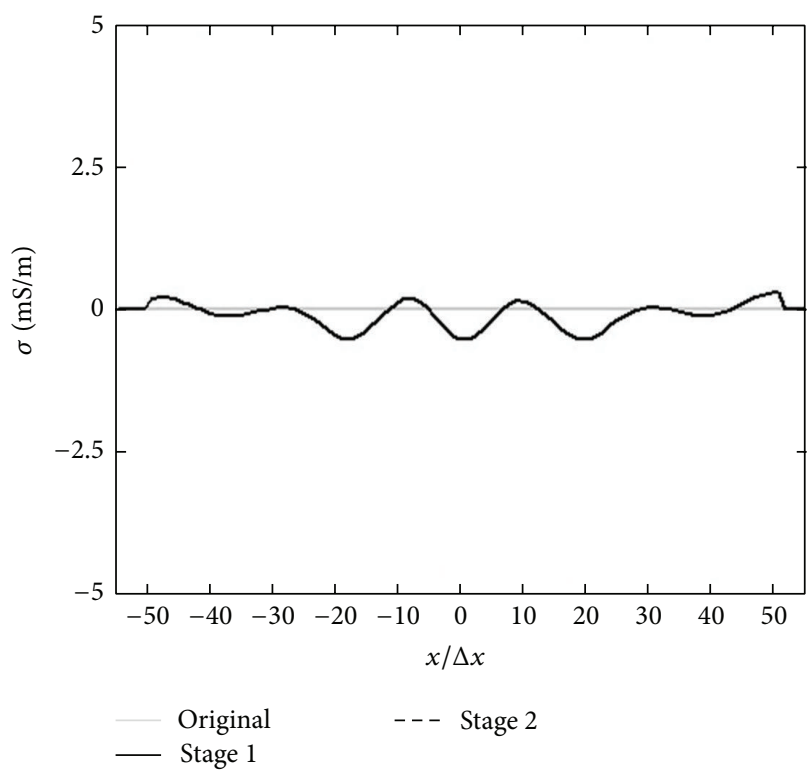

(b)

FIGURE 10: Distribution of (a) relative dielectric constant and (b) conductivity of layered targets at $z=0 ; R_{t}=0.1 \mathrm{~m}, R_{t i}=0.4 \mathrm{~m}, R_{t e}=0.5 \mathrm{~m}$, $\epsilon_{r}=2.5, \sigma=0, M=48$, and $R_{d}=1.25 \mathrm{~m}$.

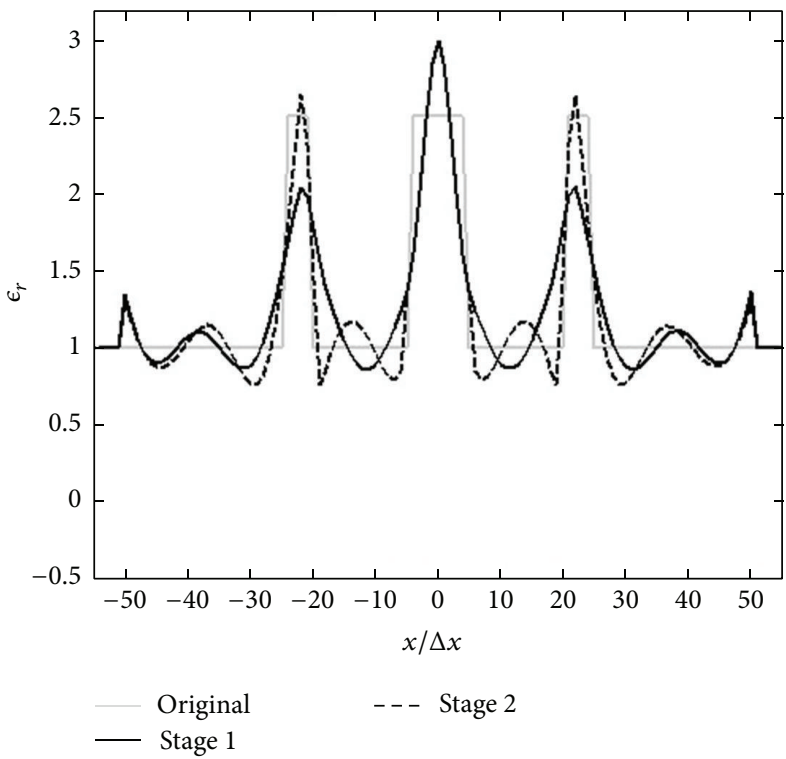

(a)

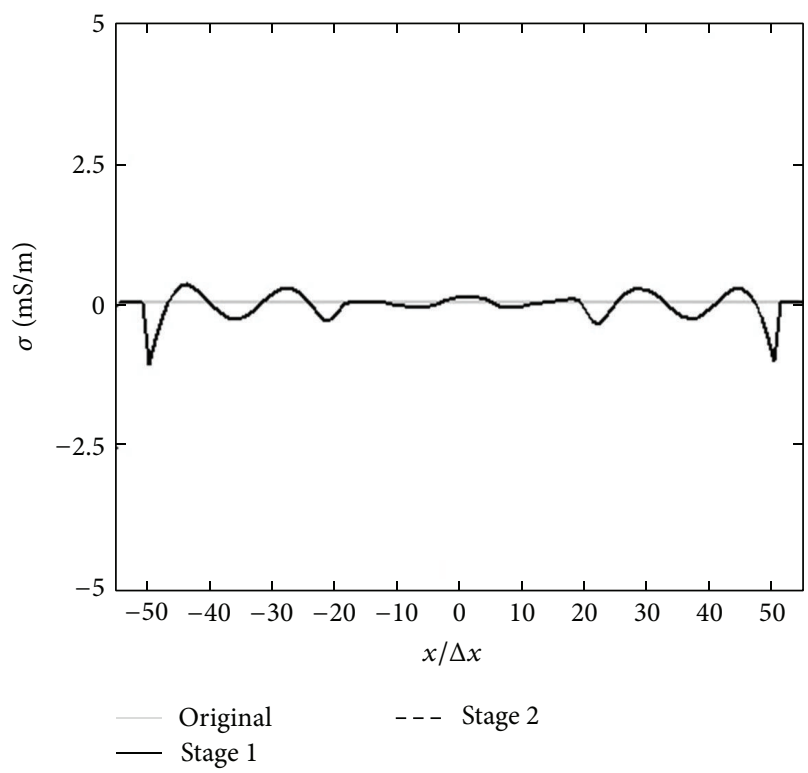

(b)

FIGURE 11: Distribution of (a) relative dielectric constant and (b) conductivity of layered targets at $z=0 ; R_{t}=0.1 \mathrm{~m}, R_{t i}=0.5 \mathrm{~m}, R_{t e}=0.6 \mathrm{~m}$, $\epsilon_{r}=2.5$ and $\sigma=0, M=48$, and $R_{d}=1.25 \mathrm{~m}$.

seriously underestimated, similar to those shown in Figures 14 and 15.

The incident waves are partially reflected at the interfaces between adjacent stairs, and smaller discontinuity of $\epsilon_{r}$ over the interfaces leads to less reflection. Hence, more incident waves can reach the internal portion of the target domain and get more information to estimate the internal permittivity.

Figure 16 shows the recovered distribution of relative dielectric constant at $z=0$, using the iterative approach. A linear profile with $\epsilon_{r, \max }=2.4$ is assumed, and $R_{t}=0.5 \mathrm{~m}$. By merging the first few external layers to the background 


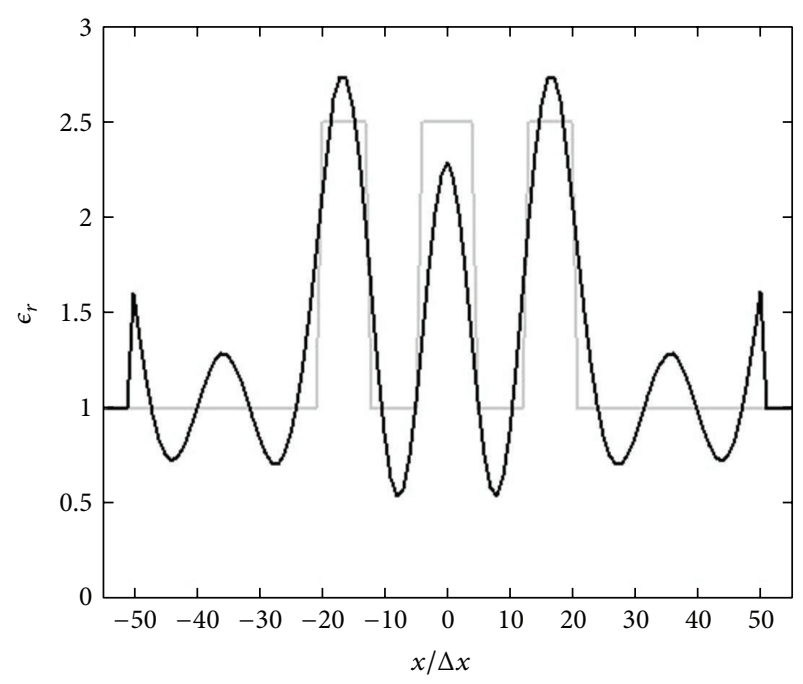

Original

- Stage 1

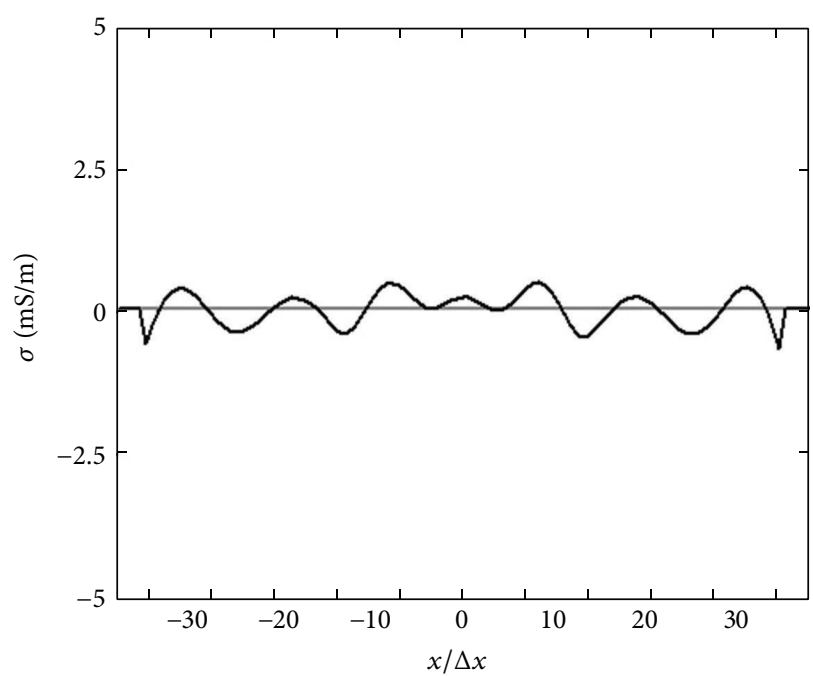

Original

Stage 1

(a)

(b)

FIGURE 12: Distribution of (a) relative dielectric constant and (b) conductivity of layered targets at $z=0 ; R_{t}=0.1 \mathrm{~m}, R_{t i}=0.3 \mathrm{~m}, R_{t e}=0.5 \mathrm{~m}$, $\epsilon_{r}=2.5, \sigma=0, M=48$, and $R_{d}=1.25 \mathrm{~m}$.

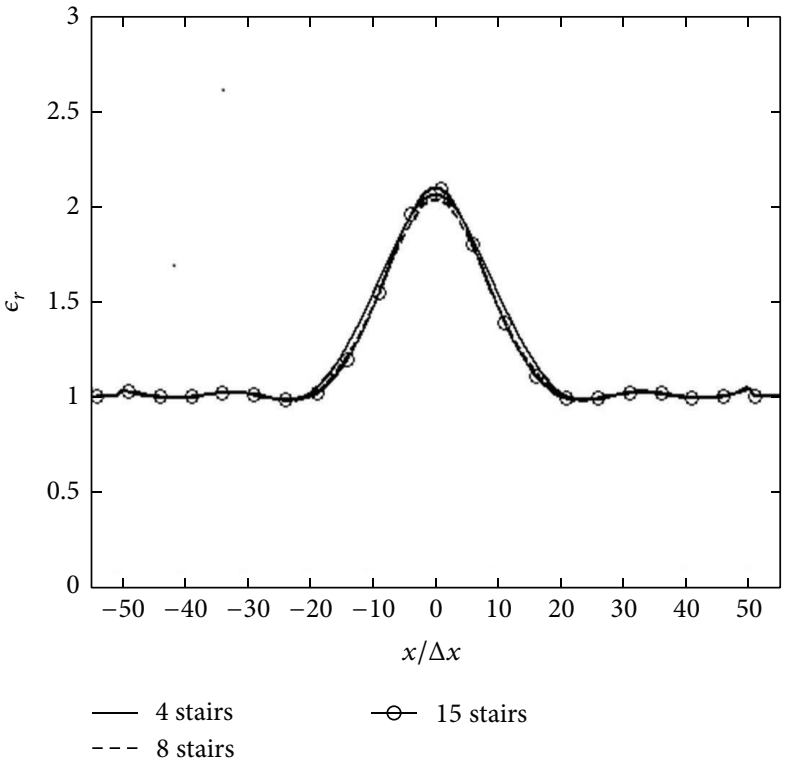

(a)

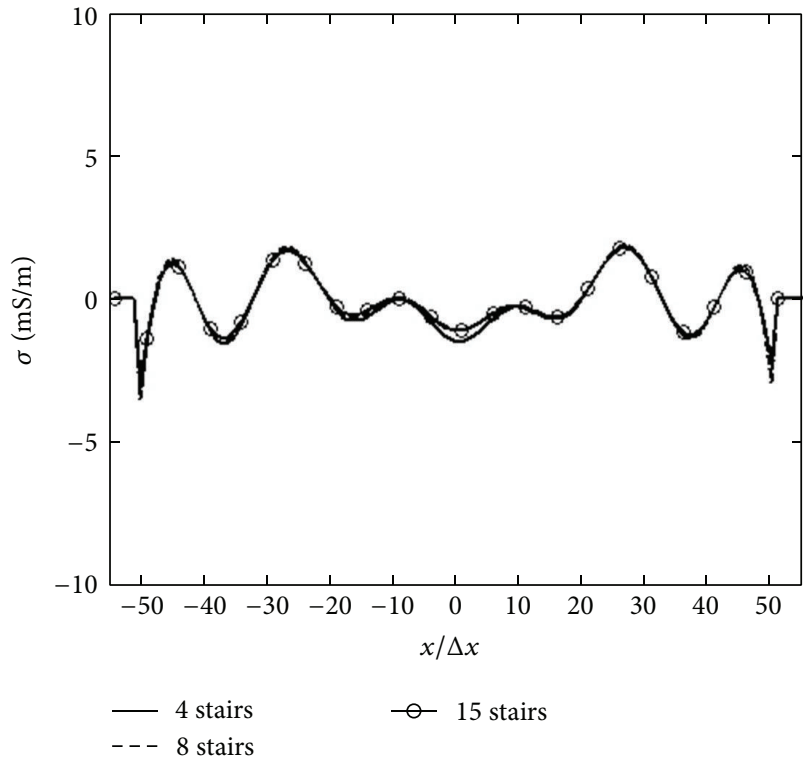

(b)

FIGURE 13: Distribution of (a) relative dielectric constant and (b) conductivity at $z=0 ; R_{t}=0.375 \mathrm{~m}$, linear profile with $\epsilon_{r \text {, max }}=2.4, \sigma=0$, $M=48$, and $R_{d}=1.25 \mathrm{~m}$.

medium in stage 2 , the internal portion is recovered more accurately. The recovered image with $0.35 \mathrm{~m}<R_{t}<0.55 \mathrm{~m}$ merged as the background medium matches the most with the original one. However, the recovered distribution at the interface between the background medium and the target becomes less accurate because the contrast function $\chi$ jumps from zero to a finite number at the interface.

In order to avoid the discontinuity of $\chi$, the permittivity at the interface estimated in stage 1 is used as the background permittivity inside the interface in stage 2 . As shown in 


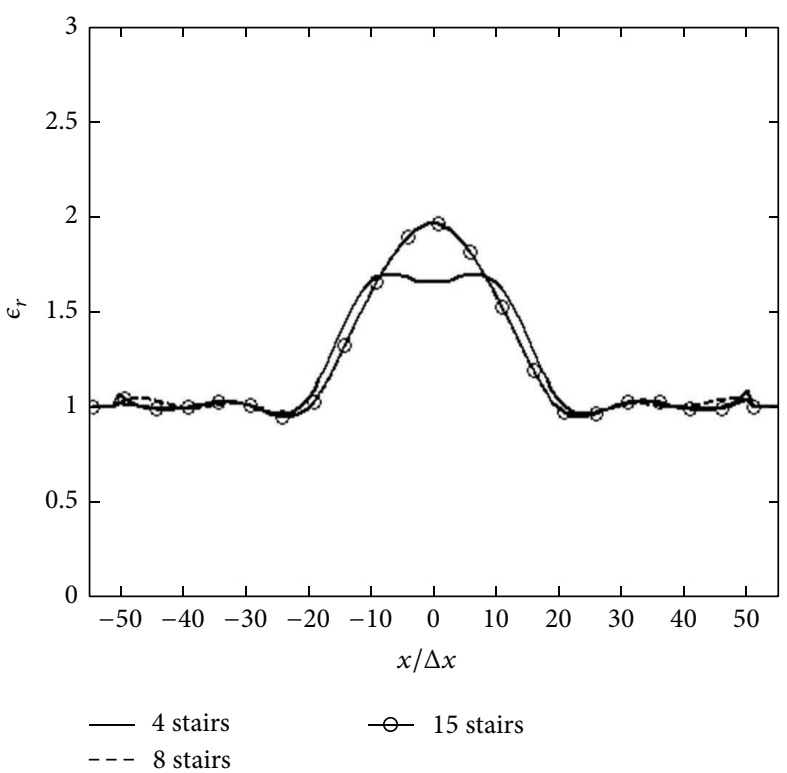

(a)

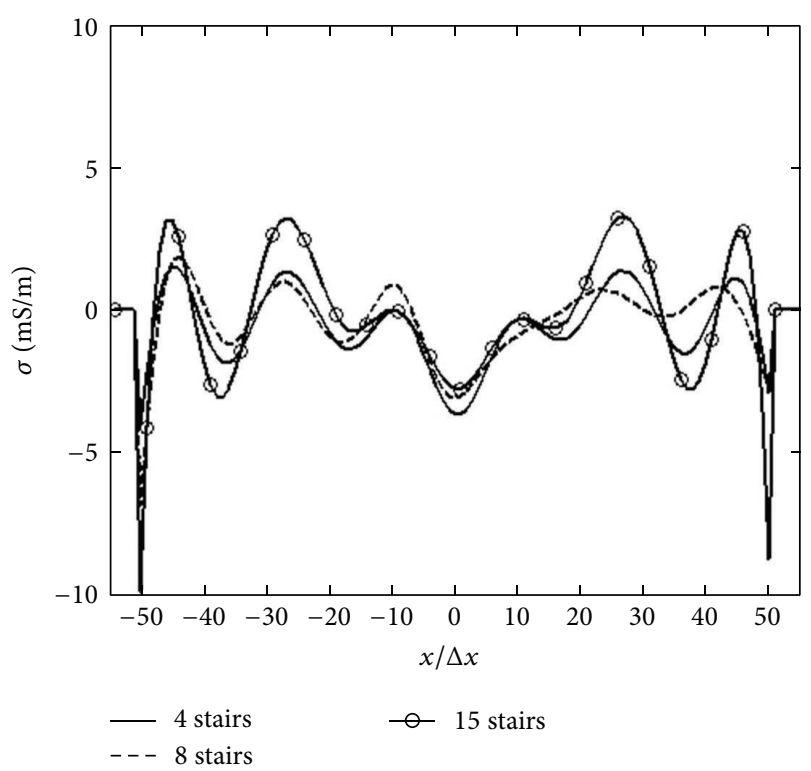

(b)

FIGURE 14: Distribution of (a) relative dielectric constant and (b) conductivity at $z=0 ; R_{t}=0.375 \mathrm{~m}$, linear profile with $\epsilon_{r, \max }=3, \sigma=0$, $M=48$, and $R_{d}=1.25 \mathrm{~m}$.

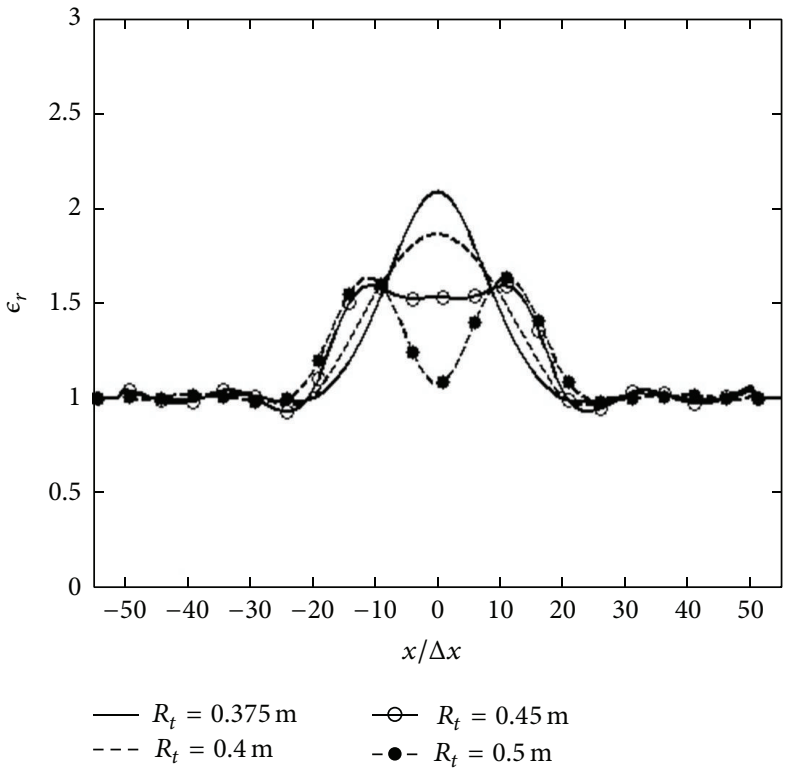

(a)

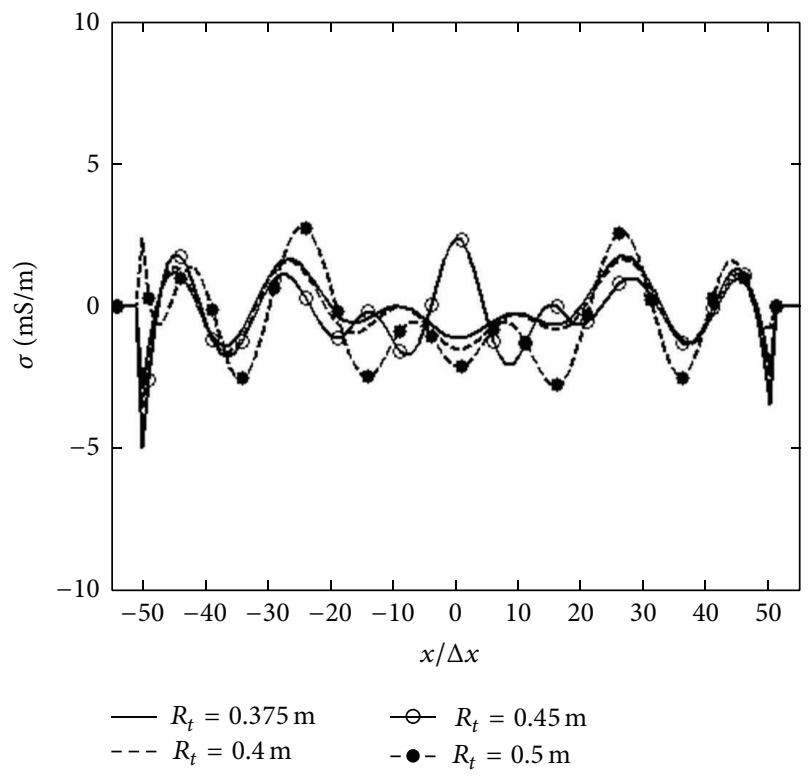

(b)

FIGURE 15: Distribution of (a) relative dielectric constant and (b) conductivity at $z=0$; linear profile with $\epsilon_{r, \max }=2.4$ and $\sigma=0$.

Figure 17, the discontinuity problem is reduced, if not completely removed. When $0.35 \mathrm{~m}<R_{t}<0.55 \mathrm{~m}$ is selected as the background, $\varepsilon_{s}$ is reduced from $16 \%$ to $15 \%$ and $\varepsilon_{\epsilon t}$ is reduced from $34 \%$ to $5 \%$ by comparing the results after stages 1 and 2.

\section{Conclusion}

An iterative approach, based on LSM and CSI method, is proposed to improve the accuracy of recovered images for multiple targets, layered targets, and targets with a continuous permittivity profile. For multiple targets, when a target is partially blocked by other targets, its permittivity tends to be underestimated, and that of the gap between targets tends to be overestimated. For layered targets, the external layers tend to be shifted inwards, especially when the gap between layers is small or the external layer is thin. For a cylinder with continuous permittivity profile, when the radius or the spatial change rate of permittivity is large, the permittivity in the internal portion tends to be underestimated. All 


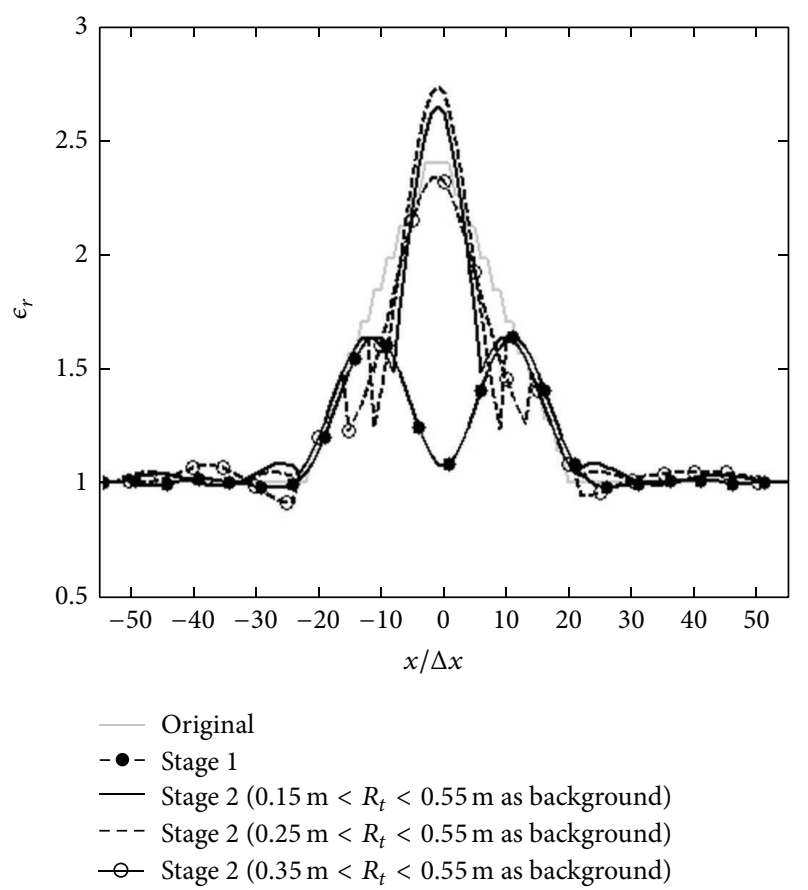

FIGURE 16: Distribution of relative dielectric constant at $z=0$; linear profile with $\epsilon_{r, \max }=2.4, \sigma=0$, and $R_{t}=0.5 \mathrm{~m}$.

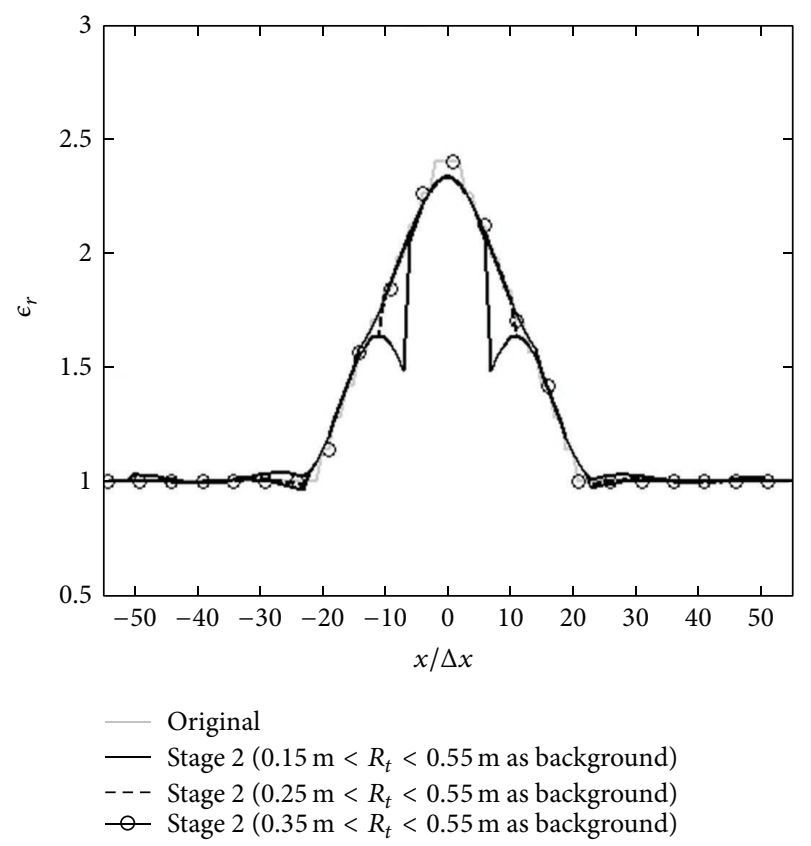

FIGURE 17: Distribution of relative dielectric constant at $z=0$; linear profile with $\epsilon_{r, \max }=2.4, \sigma=0$, and $R_{t}=0.5 \mathrm{~m}$.

these symptoms can be relieved with the proposed iterative approach, which are validated by simulations.

\section{Conflict of Interests}

The authors declare that there is no conflict of interests regarding the publication of this paper.

\section{Acknowledgments}

This work was sponsored by the Ministry of Science and Technology, Taiwan, under Contract NSC 101-2221-E-002-129 and the Ministry of Education, Taiwan, under Aim for Top University Project 103R3401-1.

\section{References}

[1] L. Crocco, I. Catapano, L. D. Donato, and T. Isernia, "The linear sampling method as a way to quantitative inverse scattering," IEEE Transactions on Antennas and Propagation, vol. 60, no. 4, pp. 1844-1853, 2012.

[2] I. Catapano, L. Crocco, and T. Isernia, "Improved sampling methods for shape reconstruction of 3-D buried targets," IEEE Transactions on Geoscience and Remote Sensing, vol. 46, no. 10, pp. 3265-3273, 2008.

[3] D. Colton, H. Haddar, and M. Piana, "The linear sampling method in inverse electromagnetic scattering theory," Inverse Problems, vol. 19, no. 6, pp. S105-S137, 2003.

[4] A. M. Hassan, M. R. Hajihashemi, and M. El-Shenawee, "Inverse scattering shape reconstruction of 3D bacteria using the level set algorithm," Progress in Electromagnetics Research B, vol. 39, pp. 39-53, 2012.

[5] M. R. Hajihashemi and M. El-Shenawee, "Level set algorithm for shape reconstruction of non-overlapping three-dimensional penetrable targets," IEEE Transactions on Geoscience and Remote Sensing, vol. 50, no. 1, pp. 75-86, 2012.

[6] C. Gilmore, A. Abubakar, W. Hu, T. M. Habashy, and P. M. van den Berg, "Microwave biomedical data inversion using the finite-difference contrast source inversion method," IEEE Transactions on Antennas and Propagation, vol. 57, no. 5, pp. 1528-1538, 2009.

[7] A. Abubakar, W. Hu, P. M. van den Berg, and T. M. Habashy, "A finite-difference contrast source inversion method," Inverse Problems, vol. 24, no. 6, Article ID 065004, 2008.

[8] P. M. van den Berg and R. E. Kleinman, "A contrast source inversion method," Inverse Problems, vol. 13, no. 6, pp. 16071620, 1997.

[9] L. Di Donato, M. Bevacqua, T. Isernia, I. Catapano, and L. Crocco, "Improved quantitative microwave tomography by exploiting the physical meaning of the Linear Sampling Method," in Proceedings of the 5th European Conference on Antennas and Propagation (EUCAP '11), pp. 3828-3831, Rome, Italy, April 2011.

[10] X. Ye, X. Chen, Y. Zhong, and R. Song, "Simultaneous reconstruction of dielectric and perfectly conducting scatterers via $\mathrm{T}$ matrix method," IEEE Transactions on Antennas and Propagation, vol. 61, no. 7, pp. 3774-3781, 2013.

[11] E. Mudry, P. C. Chaumet, K. Belkebir, and A. Sentenac, "Electromagnetic wave imaging of three-dimensional targets using a hybrid iterative inversion method," Inverse Problems, vol. 28 , no. 6, 2012.

[12] J. M. Geffrin and P. Sabouroux, "Testing inversion algorithms against experimental data," Inverse Problems, vol. 17, no. 6, pp. 1565-1571, 2001.

[13] M. Ostadrahimi, A. Zakaria, J. Lovetri, and L. Shafai, "A nearfield dual polarized (TE-TM) microwave imaging system," IEEE Transactions on Microwave Theory and Techniques, vol. 61, no. 3, pp. 1376-1384, 2013. 
[14] A. Zakaria, C. Gilmore, and J. LoVetri, "Finite-element contrast source inversion method for microwave imaging," Inverse Problems, vol. 26, no. 11, 2010.

[15] L. Pan, Y. Zhong, X. Chen, and S. P. Yeo, "Subspace-based optimization method for inverse scattering problems utilizing phaseless data," IEEE Transactions on Geoscience and Remote Sensing, vol. 49, no. 3, pp. 981-987, 2011.

[16] L. Pan, X. Chen, and S. P. Yeo, "Nondestructive evaluation of nanoscale structures: inverse scattering approach," Applied Physics A: Materials Science and Processing, vol. 101, no. 1, pp. 143-146, 2010.

[17] L. Pan, X. Chen, Y. Zhong, and S. P. Yeo, "Comparison among the variants of subspace-based optimization method for addressing inverse scattering problems transverse electric case," Journal of the Optical Society of America A, vol. 27, no. 10, pp. 2208-2215, 2010.

[18] C. Gilmore, P. Mojabi, A. Zakaria, S. Pistorius, and J. Lovetri, "On super-resolution with an experimental microwave tomography system," IEEE Antennas and Wireless Propagation Letters, vol. 9, pp. 393-396, 2010.

[19] Y. Zhong, X. Chen, and K. Agarwal, "An improved subspacebased optimization method and its implementation in solving three-dimensional inverse problems," IEEE Transactions on Geoscience and Remote Sensing, vol. 48, no. 10, pp. 3763-3768, 2010.

[20] M. D’Urso, T. Isernia, and A. F. Morabito, "On the solution of 2 - $\mathrm{d}$ inverse scattering problems via source-type integral equations," IEEE Transactions on Geoscience and Remote Sensing, vol. 48, no. 3, pp. 1186-1198, 2010. 

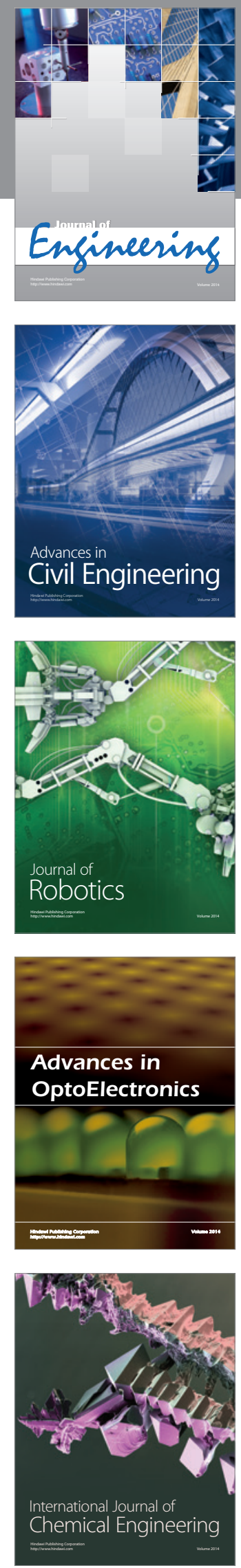

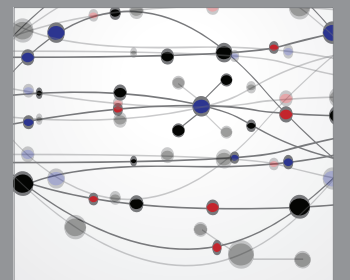

The Scientific World Journal
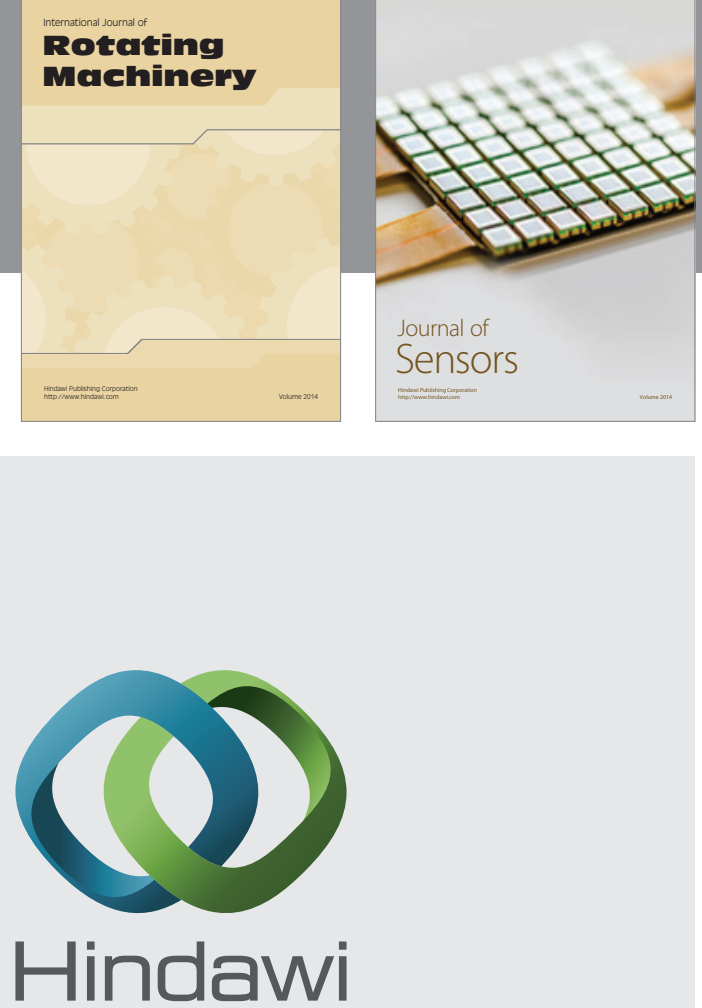

Submit your manuscripts at http://www.hindawi.com
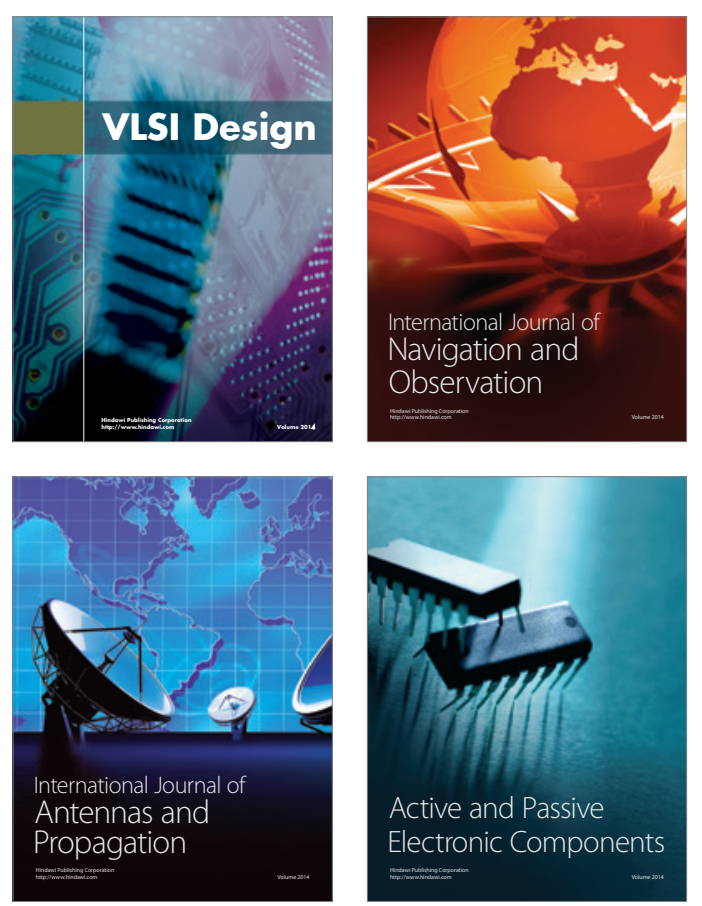
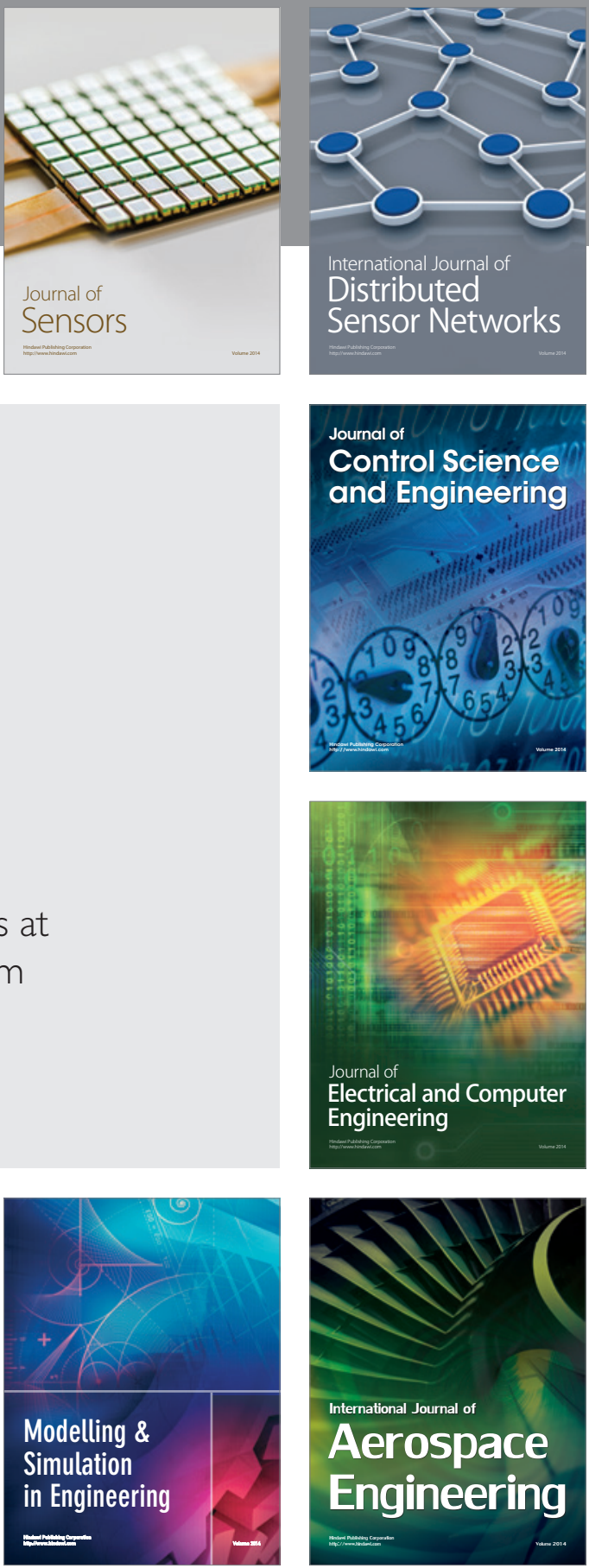

Journal of

Control Science

and Engineering
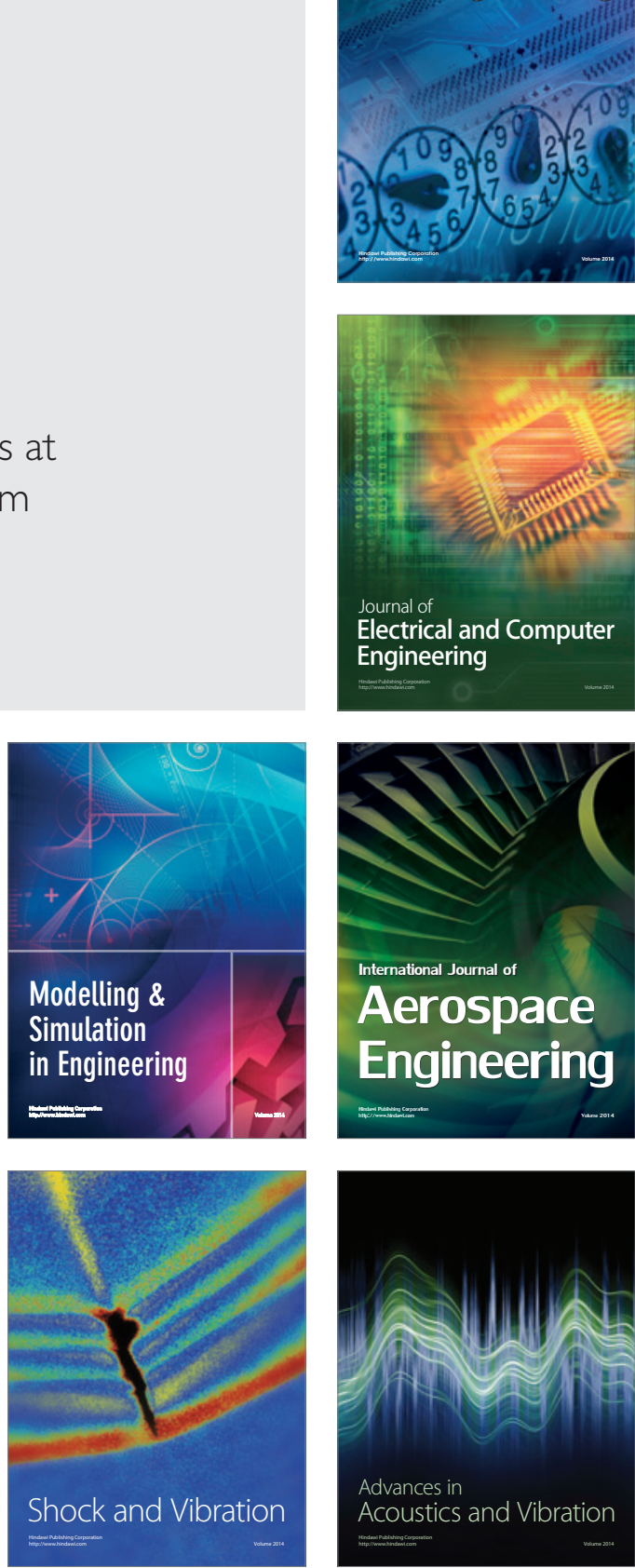